NBER WORKING PAPER SERIES

LOCAL SECTORAL SPECIALIZATION IN A WARMING WORLD

\author{
Bruno Conte \\ Klaus Desmet \\ Dávid Krisztián Nagy \\ Esteban Rossi-Hansberg \\ Working Paper 28163 \\ http://www.nber.org/papers/w28163 \\ NATIONAL BUREAU OF ECONOMIC RESEARCH \\ 1050 Massachusetts Avenue \\ Cambridge, MA 02138 \\ December 2020
}

The views expressed herein are those of the authors and do not necessarily reflect the views of the National Bureau of Economic Research.

NBER working papers are circulated for discussion and comment purposes. They have not been peer-reviewed or been subject to the review by the NBER Board of Directors that accompanies official NBER publications.

(C) 2020 by Bruno Conte, Klaus Desmet, Dávid Krisztián Nagy, and Esteban Rossi-Hansberg. All rights reserved. Short sections of text, not to exceed two paragraphs, may be quoted without explicit permission provided that full credit, including $\odot$ notice, is given to the source. 
Local Sectoral Specialization in a Warming World

Bruno Conte, Klaus Desmet, Dávid Krisztián Nagy, and Esteban Rossi-Hansberg

NBER Working Paper No. 28163

December 2020

JEL No. F18,O13,O41,Q56,R11,R12

\begin{abstract}
This paper quantitatively assesses the world's changing economic geography and sectoral specialization due to global warming. It proposes a two-sector dynamic spatial growth model that incorporates the relation between economic activity, carbon emissions, and temperature. The model is taken to the data at the $1^{\circ}$ by $1^{\circ}$ resolution for the entire world. Over a 200-year horizon, rising temperatures consistent with emissions under Representative Concentration Pathway 8.5 push people and economic activity northwards to Siberia, Canada, and Scandinavia. Compared to a world without climate change, clusters of agricultural specialization shift from Central Africa, Brazil, and India's Ganges Valley, to Central Asia, parts of China and northern Canada. Equatorial latitudes that lose agriculture specialize more in non-agriculture but, due to their persistently low productivity, lose population. By the year 2200, predicted losses in real GDP and utility are $6 \%$ and $15 \%$, respectively. Higher trade costs make adaptation through changes in sectoral specialization more costly, leading to less geographic concentration in agriculture and larger climate-induced migration.
\end{abstract}

\author{
Bruno Conte \\ Departament d'Economia i \\ d'Historia Economica \\ Universitat Autonoma de Barcelona \\ Bellaterra (Barcelona) 08193 \\ Spain \\ bruno.conte@barcelonagse.eu \\ Klaus Desmet \\ Department of Economics and Cox \\ School of Business \\ Southern Methodist University 3300 \\ Dyer, Suite 301 \\ Dallas, TX 75205 \\ and CEPR \\ and also NBER \\ kdesmet@smu.edu
}

\author{
Dávid Krisztián Nagy \\ CREI \\ Ramon Trias Fargas, 25-27 \\ 08005 Barcelona \\ Spain \\ dnagy@crei.cat \\ Esteban Rossi-Hansberg \\ Princeton University \\ Department of Economics \\ 289 Julis Romo Rabinowitz Building \\ Princeton, NJ 08544-1021 \\ and CEPR \\ and also NBER \\ erossi@princeton.edu
}




\title{
Local Sectoral Specialization in a Warming World*
}

\author{
Bruno Conte Klaus Desmet \\ $U A B$ \\ $S M U$ \\ Dávid Krisztián Nagy \\ CREI \\ Esteban Rossi-Hansberg \\ Princeton University
}

November 23, 2020

\begin{abstract}
This paper quantitatively assesses the world's changing economic geography and sectoral specialization due to global warming. It proposes a two-sector dynamic spatial growth model that incorporates the relation between economic activity, carbon emissions, and temperature. The model is taken to the data at the $1^{\circ}$ by $1^{\circ}$ resolution for the entire world. Over a 200-year horizon, rising temperatures consistent with emissions under Representative Concentration Pathway 8.5 push people and economic activity northwards to Siberia, Canada, and Scandinavia. Compared to a world without climate change, clusters of agricultural specialization shift from Central Africa, Brazil, and India's Ganges Valley, to Central Asia, parts of China and northern Canada. Equatorial latitudes that lose agriculture specialize more in nonagriculture but, due to their persistently low productivity, lose population. By the year 2200, predicted losses in real GDP and utility are $6 \%$ and $15 \%$, respectively. Higher trade costs make adaptation through changes in sectoral specialization more costly, leading to less geographic concentration in agriculture and larger climate-induced migration.
\end{abstract}

\section{Introduction}

Global warming will change the comparative advantage of regions across the world. Areas that today have ideal temperatures for agricultural production, such as parts of India, Africa, and South America, will become too hot for agriculture and will adapt by switching to other sectors. Of course, their ability to shift specialization as an adaptation mechanism depends on their productivity in other sectors, such as manufacturing and services, as well as on their ability to trade with other parts of the world. If adaptation through sectoral specialization is ineffective, regions will suffer and population will migrate elsewhere, to areas in the world where conditions are more hospitable.

Assessing the changing economic geography of a warming world therefore requires a high-resolution multi-sector dynamic spatial model that is able to evaluate the relative importance of trade and migration as adaptation mechanisms in different parts of the world. Migration and trade are costly, so incorporating realistic frictions to moving people and goods is paramount. Of course, while climate change affects the economy, the reverse is true as well. Hence, explicitly modeling the relation between economic activity, carbon emissions, and temperature is essential too.

\footnotetext{
${ }^{*}$ Conte: Department of Economics, Universitat Autònoma de Barcelona, bruno.conte@barcelonagse.eu, Desmet: Department of Economics and Cox School of Business, Southern Methodist University, kdesmet@smu.edu, Nagy: Centre de Recerca en Economia Internacional, dnagy@crei.cat, Rossi-Hansberg: Department of Economics, Princeton University, erossi@princeton.edu.
} 
Starting with the spatial dynamic model of the world economy of Desmet et al. (2018), we introduce three changes to make it amenable to assessing the spatial and sectoral impact of global warming. A first change extends the theory to multiple sectors. ${ }^{1}$

A second change allows sectoral productivity to depend on temperature. Because certain sectors, such as agriculture, are more sensitive to rising temperatures, than other sectors, such as manufacturing and services, and because different locations start off with different temperature levels, a shock to temperature translates into a local shock to comparative advantage. As such, changing specialization patterns constitute a relevant margin of adjustment to climate change. A third, and last, change follows standard integrated assessment models by explicitly introducing the feedback from the economy to the climate. Production requires energy use, which leads to emissions. Through the carbon cycle, emissions affect the atmospheric stock of carbon, translating into rising temperatures.

In any quantitative assessment of global warming, using high-quality data is essential. We use data on population, total output, agricultural output, and temperature at the $1^{\circ}$ by $1^{\circ}$ resolution for the entire world. Although the model allows for any number of sectors, we focus on just two: agriculture and a sector that combines all others, which we refer to as non-agriculture. Our baseline exercise calibrates to an increase in the carbon stock and in global temperature consistent with the predictions under Representative Concentration Pathway (RCP) 8.5 (van Vuuren et al., 2011; IPCC, 2020). This is a high-emissions pathway based on fossil-fuel-intensive economic growth, leading to a 1200 GTC increase in the stock of carbon and a $3.7^{\circ} \mathrm{C}$ global temperature increase by the end of the 21 st century. One important element in our calibration is how sensitive local temperatures are to a rise in global temperature. For example, it is well known that the poles are warming faster than the rest of the world. Due to, among others, the albedo effect and poleward energy transport, in some polar regions a one-degree increase in global temperature translates into a more than three-degree increase in local temperature. To estimate location-specific parameters that map changes in global temperature into changes in local temperature, we use predicted local and global temperatures between 2000 and 2100 from the Intergovernmental Panel on Climate Change (IPCC). Another key element in our calibration is how sensitive agricultural and non-agricultural productivities are to temperature. For agriculture, we base ourselves on established estimates of the relation between temperature and crop yields from the agronomy literature, whereas for non-agriculture we use the model-predicted estimates of nonagricultural productivity to estimate its relation to temperature.

After calibrating the model, we simulate the model forward for 200 years. The baseline simulation assumes that frictions to moving people and moving goods remain unchanged at current levels. Our main results can be summarized as follows. First, while in the next 200 years many of the world's densest and richest regions continue to be dense and rich, climate change does have an impact. In terms of population, Scandinavia, northern Canada and Siberia gain, whereas the Arabian peninsula, northern India, North Africa, Brazil and Central America lose. In terms of income per capita, patterns are similar, though losses are more widespread, essentially spanning all latitudes comprised between southern Africa and southern Europe. One exception are coastal areas that display greater resilience.

Second, when considering sectoral specialization, agriculture becomes spatially more concentrated. While this move toward greater geographic concentration happens independently of climate change, rising

\footnotetext{
${ }^{1}$ We take preferences to be Cobb-Douglas across sectors. While this implies constant expenditure shares, we still find a declining agricultural employment share because of the climate-induced relocation of agriculture toward land-abundant areas. In many models of long-run development and structural transformation, the falling agricultural employment share is generated by either nonhomothetic preferences or an elasticity of substitution between agriculture and other sectors of less than one (e.g., Uy et al., 2013; Herrendorf et al., 2014; Świecki, 2017).
} 
temperatures affect where the increased concentration occurs. In the absence of climate change, clusters of agricultural specialization can be found in South America, sub-Saharan Africa, and India's Ganges Valley. With rising temperature, these clusters shift to Central Asia, China, and Canada. In contrast to what one might hope, most of the regions that lose agriculture do not become thriving non-agricultural powerhouses. This is especially true in developing countries that start off with low-tech manufacturing and services.

Third, in the aggregate, by the year 2200 global warming leads to a $6 \%$ decrease in income per capita and a $15 \%$ decrease in utility. The larger drop in utility is related to climate change pushing people northward to areas with worse amenities, such as Siberia or northern Canada. How do the different sectors perform in the aggregate? Although agriculture is more sensitive to climate change than non-agriculture, we find that rising temperatures increase productivity growth in agriculture and decrease productivity growth in non-agriculture. Warmer temperatures push agriculture to regions, such as Central Asia, that initially suffered from a large temperature penalty. With global warming, these regions benefit from relatively high agricultural productivity.

To explore the role of trade, we conduct a number of counterfactual exercises with higher and lower trade costs. We find that higher trade costs lead to greater climate-induced movements of people. This indicates that trade and migration are substitutes: higher trade costs limit the scope of locally adjusting to a climate shock by changing specialization. This makes adjusting through migration relatively more attractive. These larger spatial changes when trade is more costly are also present when analyzing real GDP per capita.

When considering patterns of specialization, higher trade costs limit the spatial concentration of agriculture. Because goods are sourced from locations that are closer by when trade is more costly, agriculture in the year 2200 is spatially more dispersed under high trade costs than under low trade costs. In the aggregate, by the year 2200 climate-induced losses in global income per capita are higher under low trade costs than under high trade costs, though that difference is reversed by the year 2400. One might have expected that higher trade costs would lead to larger climate-induced losses throughout. After all, with higher trade costs, there is less scope to respond to the sector-specific effects of global warming by changing specialization. However, higher trade costs lead to a greater shift of population and economic activity to high-productivity places in the U.S., Europe, and Japan, that are relatively less affected by the growing temperatures.

Our paper is related to a growing literature aimed at quantifying the economic effects of climate change across the globe. Nordhaus $(1993,2008,2010)$ pioneered the development of integrated assessment models that incorporate the main insights of climate science into economic growth models. Other examples of integrated assessment models that build on standard quantitative macro frameworks include Golosov et al. (2014) and Hassler et al. (2016). While many of these models have only one region, some allow for multiple regions, and are thus able to evaluate how climate change affects different regions differently. However, with only a handful of regions, they are unable to capture the rich spatial heterogeneity of the effects of climate change. In addition, these models do not include trade and migration in the economy's response to global warming.

In recent years a burgeoning literature has developed high-resolution spatial models: most are static (Allen and Arkolakis, 2014), and only a few are dynamic (Desmet and Rossi-Hansberg, 2014; Desmet et al., 2018; Caliendo et al., 2019). ${ }^{2}$ Needless to say, in the context of global warming, dynamics are of the essence, because of the slow-moving nature of climate change. Some of these spatial dynamic models have already

\footnotetext{
${ }^{2}$ See Redding and Rossi-Hansberg (2017) for a review of the quantitative spatial literature.
} 
been applied to evaluate the economic impact of climate change. Desmet and Rossi-Hansberg (2015) assess the spatial dynamic effect of global warming in a two-sector model with one-dimensional space. While a one-dimensional model is a reasonable simplification - only a small fraction of the variance in temperature occurs within latitudes - it fails to capture relevant differences between, for example, coastal areas and more inland regions. Using a two-dimensional spatial growth model that captures the world's true geography, Desmet et al. (2020) carry out a quantitative assessment of rising sea levels. Another relevant paper is Balboni (2019) who analyzes the welfare effects of large coastal infrastructure investments in Vietnam in a dynamic spatial model that takes into account future inundation.

In a contemporaneous and related contribution, Cruz and Rossi-Hansberg (2020) propose a similar dynamic spatial model to evaluate the geography of the economic costs of global warming. They add a number of features that we abstract from here. In particular they incorporate the effect of changes in temperature on amenities and fertility, and model the choice to use and produce clean and carbon based energy. Importantly, they limit their analysis to one aggregate sector. Hence, although richer in some dimensions, they cannot study the role of specialization as an adaptation mechanism to global warming, which is our central goal.

A key contribution of spatial dynamic models is the explicit treatment of trade and migration. Since climate change affects some locations more negatively than others, migration is an adaptation strategy. And because not all sectors are impacted in the same way, so is trade. ${ }^{3}$ For example, Desmet et al. (2020) find that the loss in real GDP due to coastal flooding in the year 2200 drops from $4.5 \%$ to $0.11 \%$ when incorporating the dynamic response of migration. In a related paper, Burzyński et al. (2019) predict that climate change will induce the displacement of 200 to 300 million people over the course of the 21st century, though only $20 \%$ will involve cross-boarder migration. In another recent evaluation, Benveniste et al. (2020) find substantially smaller numbers, estimating excess climate-induced cross-border migration flows in the year 2100 of 75,000. In our paper, we also focus on the importance of mobility, and highlight that trade and migration may be substitutes in their response to climate shocks.

A large part of the literature on climate change deals with policies aimed at mitigating global warming. In fact, many integrated assessment models seek to quantify the optimal carbon tax (Nordhaus, 2010; Golosov et al., 2014; Hassler et al., 2016, 2018). Other papers analyze the use of different policies to promote the transition to clean energy (Acemoglu et al., 2012, 2016). While we do not focus on mitigation and energy transition, our paper does feature endogenous innovation. As a result, energy use per unit of production declines over time. In their related framework, Cruz and Rossi-Hansberg (2020) study carbon taxes, clean energy subsidies, and abatement policies.

The rest of the paper is organized as follows. Section 2 develops the model. Section 3 describes the data and quantification of the model. Section 4 reports the main findings and Section 5 concludes. Appendix A includes details on how to solve and invert the model to obtain local amenities and productivities by sector.

\section{Model}

Our starting point is the high-resolution dynamic spatial model of the world economy with trade and migration frictions of Desmet et al. (2018). To assess the economic impact of climate change, we extend this model

\footnotetext{
${ }^{3}$ Without using a spatial dynamic framework, other relevant papers that have analyzed the effect of climate change on comparative advantage include Costinot et al. (2016) and Conte (2020) who emphasize the importance of crop switching, as well as Nath (2020), who shows that subsistence food requirements may keep more people employed in agriculture in some of the areas that are hardest hit by climate change.
} 
in three ways. First, we allow for multiple sectors. To be precise, the economy consists of $I$ sectors, indexed by $i=1,2, \ldots, I$, with each sector producing a continuum of goods $\omega \in[0,1]$. Agents' utility is CES across goods within each sector and Cobb-Douglas across sectors. Labor is freely mobile across sectors. Second, at each location, sectoral productivity levels depend on the location's temperature. Third, each sector uses energy to produce. Energy is freely tradable and is supplied by a resource extraction sector that operates under decreasing returns. Energy use contributes to $\mathrm{CO}_{2}$ emissions, and hence to the atmospheric stock of carbon, which affects temperature at every location. As a result, economic activity depends on temperature, and temperature depends on economic activity. We now proceed to describing the model in further detail. Inevitably, part of the description draws on Desmet et al. (2018).

\subsection{Model Setup}

Endowments and preferences. The world economy occupies a two-dimensional surface $S$, where a location is defined as a point $r \in S$. Location $r$ has land density $H(r)$, and there are $\bar{L}$ agents in the world economy, each supplying one unit of labor. An agent $j$ who lives in location $r \in S$ in period $t$ with a history of having resided in $\left\{r_{0}, \ldots, r_{t-1}\right\}$ enjoys utility

$$
U_{t}^{j}\left(r_{0}, \ldots, r_{t-1}, r\right)=\bar{\chi} a_{t}(r) \prod_{i=1}^{I}\left[\int_{0}^{1} c_{i t}^{\omega}(r)^{\rho} d \omega\right]^{\frac{\chi_{i}}{\rho}} \varepsilon_{t}^{j}(r) \prod_{s=1}^{t} m\left(r_{s-1}, r_{s}\right)^{-1}
$$

in period $t$, where $a_{t}(r)$ denotes local amenities, $c_{i t}^{\omega}(r)$ is the consumption of variety $\omega$ of good $i, 1 /(1-\rho)$ is the elasticity of substitution between different varieties of the same good, $\chi_{i}$ is the share of good $i$ in the agent's expenditure, $\varepsilon_{t}^{j}(r)$ is a location preference shock drawn from a Fréchet distribution with shape parameter $1 / \Omega, m\left(r_{s-1}, r_{s}\right)$ is the cost of moving from $r_{s-1}$ in period $s-1$ to $r_{s}$ in period $s$, and $\bar{\chi}=\prod_{i=1}^{I} \chi_{i}^{-\chi_{i}}$ is a constant that simplifies subsequent expressions. Agents discount future utility using the discount factor $\beta$.

Local amenities at location $r$ suffer from congestion and take the form:

$$
a_{t}(r)=\bar{a}(r)\left(\frac{\bar{L}_{t}(r)}{H(r)}\right)^{-\lambda}
$$

where $\bar{a}(r)$ denotes location $r$ 's fundamental amenity, and $\left(\bar{L}_{t}(r) / H(r)\right)^{-\lambda}$ represents a dispersion or congestion force coming from local population density (i.e., local population $\bar{L}_{t}(r)$ divided by land). The greater the value of $\lambda$, the stronger the dispersion force. In addition to the effect of density on amenities, there is another dispersion force coming from the preference shocks: a higher value of $\Omega$ implies greater taste heterogeneity, and hence a stronger incentive to spatially disperse.

The cost of moving from $r$ to $s$ is the product of an origin-specific cost, $m_{1}(r)$, and a destinationspecific cost, $m_{2}(s)$, so that $m(r, s)=m_{1}(r) m_{2}(s)$. Remaining in the same place is costless, and so $m(r, r)=m_{1}(r) m_{2}(r)=1$. This implies that the cost of leaving a location is the inverse of the cost of entering that location, i.e., $m_{2}(r)=m_{1}(r)^{-1}$. As a result, the permanent utility flow cost paid by an immigrant who enters $s$ is compensated by a permanent utility flow benefit of the same magnitude when leaving $s$. Migrants therefore only pay the flow utility moving cost while residing in the host location, making any decision to migrate reversible. This simplifies an agent's forward-looking migration decision to a static decision.

In addition to earning income from work, $w_{t}(r)$, an agent residing in $r$ at time $t$ gets a proportional 
share of local land rents, $R_{t}(r) H(r) / \bar{L}_{t}(r)$, as well as a proportional share of global profits from the resource extraction sector, $\Pi_{t} / \bar{L}$. Following Desmet et al. (2018), we can show that the number of people choosing to live in $r$ in period $t, \bar{L}_{t}(r)$, is given by

$$
\bar{L}_{t}(r)=\frac{u_{t}(r)^{1 / \Omega} m_{2}(r)^{-1 / \Omega}}{\int_{S} u_{t}(s)^{1 / \Omega} m_{2}(s)^{-1 / \Omega} d s} \bar{L},
$$

where

$$
u_{t}(r)=a_{t}(r) \frac{w_{t}(r)+\prod_{t} / \bar{L}+R_{t}(r) H(r) / \bar{L}_{t}(r)}{\prod_{i=1}^{I} P_{i t}(r)^{\chi_{i}}}
$$

and $P_{i t}(r)$ denotes the ideal price index of sector $i$, defined as

$$
P_{i t}(r)=\left[\int_{0}^{1} p_{i t}^{\omega}(r)^{\frac{\rho}{\rho-1}} d \omega\right]^{\frac{\rho-1}{\rho}},
$$

where $p_{i t}^{\omega}(r)$ is the price of variety $\omega$ at $r$.

Production of varieties. The representative firm producing variety $\omega$ in sector $i$ in location $r$ at time $t$ faces the constant returns production function

$$
q_{i t}^{\omega}(r)=L_{\phi, i t}^{\omega}(r)^{\gamma_{i}} z_{i t}^{\omega}(r) L_{i t}^{\omega}(r)^{\mu_{i}} E_{i t}^{\omega}(r)^{\sigma_{i}} H_{i t}^{\omega}(r)^{1-\gamma_{i}-\mu_{i}-\sigma_{i}}
$$

where $q_{i t}^{\omega}(r)$ denotes the firm's output, $L_{\phi, i t}^{\omega}(r)$ denotes the amount of labor hired by the firm to innovate, $L_{i t}^{\omega}(r)$ is the amount of labor hired to produce, $E_{i t}^{\omega}(r)$ is energy use, $H_{i t}^{\omega}(r)$ is the use of land, and $z_{i t}^{\omega}(r)$ is an idiosyncratic productivity shifter.

We assume that the idiosyncratic productivity shifter $z_{i t}^{\omega}(r)$ is i.i.d. across varieties, locations and time, drawn from a Fréchet distribution with c.d.f.

$$
\operatorname{Pr}\left[z_{i t}^{\omega}(r) \leq z\right]=e^{-\left(Z_{i t}(r) / z\right)^{\theta}}
$$

where $\theta>0$. By the properties of the Fréchet distribution, $Z_{i t}(r)$ is the average idiosyncratic productivity of varieties of good $i$ in location $r$. This average productivity depends on fundamental productivity, temperature, and agglomeration economies,

$$
Z_{i t}(r)=\tau_{i t}(r) g_{i}\left(T_{t}(r)\right)\left(\frac{\bar{L}_{i t}(r)}{H_{i t}(r)}\right)^{\alpha_{i}}
$$

where $\tau_{i t}(r)$ denotes the fundamental productivity of good $i$ in location $r$ at time $t, g_{i}(\cdot)$ is a temperature discount factor on the productivity of good $i, T_{t}(r)$ denotes temperature in $r$ at time $t$, and $\left(\bar{L}_{i t}(r) / H_{i t}(r)\right)^{\alpha}$ represent agglomeration forces that depend on local density in sector $i$, defined as total sectoral employment $\bar{L}_{i t}(r)=L_{\phi, i t}(r)+L_{i t}(r)$ divided by sectoral land use $H_{i t}(r)$. The greater the exogenous parameter $\alpha_{i}$, the stronger the agglomeration forces.

Across periods, a location's fundamental productivity in sector $i$ evolves according to equation

$$
\tau_{i t}(r)=L_{\phi, i, t-1}(r)^{\gamma_{i}}\left[\int_{S} e^{-\aleph d i s t(r, s)} \tau_{i, t-1}(s) d s\right]^{1-\delta} \tau_{i, t-1}(r)^{\delta},
$$

where $L_{\phi, i, t-1}(r)$ denotes the total amount of innovation labor hired in sector $i$ at time $t-1$, and $\operatorname{dist}(r, s)$ 
denotes the geographic distance between locations $r$ and $s$. As such, a location's fundamental productivity in sector $i$ depends on local past sectoral innovation, local past sectoral productivity, and the spatial diffusion of past sectoral productivity from all other locations. Spatial diffusion is essential to avoid excessive spatial concentration over time.

We assume that the sector-specific temperature discount factor is bell-shaped in temperature, so

$$
g_{i}\left(T_{t}(r)\right)=\exp \left[-\frac{1}{2}\left(\frac{T_{t}(r)-g_{i}^{o p t}}{g_{i}^{v a r}}\right)^{2}\right]
$$

where $g_{i}^{\text {opt }}$ denotes the optimal temperature in sector $i$, and $g_{i}^{\text {var }}$ is a parameter that determines the variance of the bell-shaped relationship between temperature and productivity in sector $i$. Note that the discount factor equals one at the optimal temperature but is below one at any other temperature.

Firms are perfectly competitive. Taking all prices as given, a firm producing variety $\omega$ of good $i$ chooses its inputs $L_{\phi, i t}^{\omega}(r), L_{i t}^{\omega}(r), E_{i t}^{\omega}(r)$ and $H_{i t}^{\omega}(r)$, subject to production function (6), to maximize its static profits

$$
p_{i t}^{\omega}(r, r) q_{i t}^{\omega}(r)-w_{t}(r)\left[L_{\phi, i t}^{\omega}(r)+L_{i t}^{\omega}(r)\right]-e_{t} E_{i t}^{\omega}(r)-R_{t}(r) H_{i t}^{\omega}(r),
$$

where $e_{t}$ denotes the global price of energy and $p_{i t}^{\omega}(r, r)$ is the price of variety $\omega$ of good $i$ produced and sold in $r$. The reason why a firm maximizes its static profits is because we assume that land markets are competitive and that any local investment in innovation becomes available to all potential entrants next period. Then, all future gains from innovation will be reflected in the value of the fixed factor, namely, land. Because a firm understands that its investments in innovation will yield zero profits in the future, its dynamic profit maximization decision simplifies to a static profit maximization decision. ${ }^{4}$

Let $\bar{L}_{i t}^{\omega}(r)$ denote the total labor used by the firm, that is,

$$
\bar{L}_{i t}^{\omega}(r)=L_{\phi, i t}^{\omega}(r)+L_{i t}^{\omega}(r)
$$

Integrating the first-order conditions of the firm's maximization problem across goods yields relationships between the sector-level use of factors and total sectoral employment, namely,

$$
\begin{gathered}
L_{\phi, i t}(r)=\frac{\gamma_{i}}{\gamma_{i}+\mu_{i}} \bar{L}_{i t}(r), \\
L_{i t}(r)=\frac{\mu_{i}}{\gamma_{i}+\mu_{i}} \bar{L}_{i t}(r), \\
E_{i t}(r)=\frac{\sigma_{i}}{\gamma_{i}+\mu_{i}} \frac{w_{t}(r)}{e_{t}} \bar{L}_{i t}(r), \\
H_{i t}(r)=\frac{1-\gamma_{i}-\mu_{i}-\sigma_{i}}{\gamma_{i}+\mu_{i}} \frac{w_{t}(r)}{R_{t}(r)} \bar{L}_{i t}(r) .
\end{gathered}
$$

Rearranging (16) and summing across sectors relates total land rents to wages and sectoral employment levels,

$$
R_{t}(r) H(r)=w_{t}(r) \sum_{i=1}^{I} \frac{1-\gamma_{i}-\mu_{i}-\sigma_{i}}{\gamma_{i}+\mu_{i}} \bar{L}_{i t}(r) .
$$

In each period we normalize all nominal variables by average world wages. Hence, only real variables can be

\footnotetext{
${ }^{4}$ See Desmet and Rossi-Hansberg (2014) and Desmet et al. (2018) for a more detailed description of this argument.
} 
meaningfully compared over time.

Production of energy. The world supply of energy is exogenously given by

$$
E_{t}=e_{t}^{\varphi}
$$

where $\varphi \in(0,1) .{ }^{5}$ We abstract from the costs of resource extraction, which implies that profits made in the resource extraction sector equal revenues. ${ }^{6}$ Thus,

$$
\Pi_{t}=e_{t} E_{t}=e_{t}^{1+\varphi} .
$$

Carbon cycle and the evolution of temperature. Emissions from production affect the carbon stock in the atmosphere, which in turn affects temperature. The carbon cycle determines the relation between emissions and the stock of carbon. We follow Desmet and Rossi-Hansberg (2015) in assuming a carbon cycle in the spirit of Nordhaus (2010), with the carbon stock gradually decaying over time. More specifically, the stock of carbon in period $t, K_{t}$, is given by

$$
K_{t}=\varepsilon_{1} K_{t-1}+\varepsilon_{2} E_{t-1},
$$

where $\varepsilon_{1} \leq 1$ determines how the carbon stock decays and $\varepsilon_{2}$ determines the relation between energy and carbon emissions. Note that if we were to set $\varepsilon_{1}=1$ and $K_{0}=0$, then the carbon stock is equal to cumulative emissions. ${ }^{7}$ Global temperature $T_{t}$ at time $t$ then evolves with the carbon stock according to

$$
T_{t}=T_{t-1}+\nu\left(K_{t}-K_{t-1}\right)
$$

where $\nu>0$.

The rise in temperature due to global warming is not expected to be homogeneous across space. We allow for a location-specific linear relation between changes in local temperatures and changes in global temperature as in Stocker et al. (2013). Hence,

$$
T_{t}(r)=T_{t-1}(r)+\left(T_{t}-T_{t-1}\right) \xi(r),
$$

where $\xi(r)$ are the location-specific down-scaling factors that map changes in global temperature into local temperatures.

\subsection{Equilibrium}

Prices and export shares. Perfect competition implies that the price of each variety is equal to the marginal cost of production,

$$
p_{i t}^{\omega}(r, r)=\frac{m c_{i t}(r)}{z_{i t}^{\omega}(r)},
$$

\footnotetext{
${ }^{5}$ In principle, we could allow for a supply intercept different from one. However, we can always measure energy in units such that this intercept equals one.

${ }^{6}$ See Cruz and Rossi-Hansberg (2020) for an alternative formulation in which the cost of extraction depends on the cumulative amount of carbon used in the past.

${ }^{7}$ Work by Allen et al. (2009) and Matthews et al. (2009) suggests that this is a reasonable simplification.
} 
where

$$
m c_{i t}(r)=\gamma_{i}^{-\gamma_{i}} \mu_{i}^{-\mu_{i}} \sigma_{i}^{-\sigma_{i}}\left(1-\gamma_{i}-\mu_{i}-\sigma_{i}\right)^{\gamma_{i}+\mu_{i}+\sigma_{i}-1} w_{t}(r)^{\gamma_{i}+\mu_{i}} e_{t}^{\sigma_{i}} R_{t}(r)^{1-\gamma_{i}-\mu_{i}-\sigma_{i}} .
$$

Trade across locations is costly. Let $\varsigma(s, r)$ denote the iceberg shipping cost from $r$ to $s$. Then, the price of a variety produced in $r$ and sold is $s$ is $p_{i t}^{\omega}(s, r)=\varsigma(s, r) p_{i t}^{\omega}(r, r)$.

Equation (23), the Fréchet distribution of idiosyncratic productivities, and the iceberg nature of shipping costs guarantee that prices in any location are also distributed Fréchet. Using the standard techniques of Eaton and Kortum (2002), we can write the spending of location $s$ on sector- $i$ varieties of location $r$ relative to the total spending of location $s$ on sector- $i$ varieties as

$$
\pi_{i t}(s, r)=\frac{Z_{i t}(r)^{\theta}\left[m c_{i t}(r) \varsigma(s, r)\right]^{-\theta}}{\int_{S} Z_{i t}(u)^{\theta}\left[m c_{i t}(u) \varsigma(s, u)\right]^{-\theta} d u} .
$$

One can also obtain the price index of sector $i$ at location $s$ as

$$
P_{i t}(s)=\bar{p}\left[\int_{S} Z_{i t}(r)^{\theta}\left[m c_{i t}(r) \varsigma(s, r)\right]^{-\theta} d r\right]^{-\frac{1}{\theta}}
$$

where $\bar{p}=\Gamma\left(1-\frac{\rho}{(1-\rho) \theta}\right)^{-\frac{1-\rho}{\rho}}$. Using (8), (16) and (24) allows us to rewrite equation (26) as

$$
P_{i t}(s)^{-\theta}=\kappa_{i} e_{t}^{-\sigma_{i} \theta} \int_{S} \tau_{i t}(r)^{\theta} g_{i}\left(T_{t}(r)\right)^{\theta} w_{t}(r)^{-\left(\alpha_{i}+\gamma_{i}+\mu_{i}\right) \theta} R_{t}(r)^{\left(\alpha_{i}+\gamma_{i}+\mu_{i}+\sigma_{i}-1\right) \theta} \varsigma(s, r)^{-\theta} d r
$$

where $\kappa_{i}=\bar{p}^{-\theta} \gamma_{i}^{\gamma_{i} \theta} \mu_{i}^{\mu_{i} \theta} \sigma_{i}^{\sigma_{i} \theta}\left(\gamma_{i}+\mu_{i}\right)^{\alpha_{i} \theta}\left(1-\gamma_{i}-\mu_{i}-\sigma_{i}\right)^{\left(1-\alpha_{i}-\gamma_{i}-\mu_{i}-\sigma_{i}\right) \theta}$.

Market clearing. Market clearing in sector $i$ implies that the revenue of firms producing varieties of good $i$ at any location $r, \frac{1}{\gamma_{i}+\mu_{i}} w_{t}(r) \bar{L}_{i t}(r)$, equals total spending on these varieties in the entire world, namely,

$$
\begin{aligned}
\frac{1}{\gamma_{i}+\mu_{i}} w_{t}(r) \bar{L}_{i t}(r)= & \chi_{i} \int_{S} \pi_{i t}(s, r)\left[\left(w_{t}(s)+\frac{\Pi_{t}}{\bar{L}}\right) \bar{L}_{t}(s)+R_{t}(s) H(s)\right] d s \\
= & \chi_{i} \kappa_{i} e_{t}^{-\sigma_{i} \theta} \tau_{i t}(r)^{\theta} g_{i}\left(T_{t}(r)\right)^{\theta} w_{t}(r)^{-\left(\alpha_{i}+\gamma_{i}+\mu_{i}\right) \theta} R_{t}(r)^{\left(\alpha_{i}+\gamma_{i}+\mu_{i}+\sigma_{i}-1\right) \theta} . \\
& \int_{S} P_{i t}(s)^{\theta}\left[\left(w_{t}(s)+\frac{\Pi_{t}}{\bar{L}}\right) \bar{L}_{t}(s)+R_{t}(s) H(s)\right] \varsigma(s, r)^{-\theta} d s .
\end{aligned}
$$

Worldwide market clearing for energy implies that

$$
e_{t}=\left[\sum_{i=1}^{I} \frac{\sigma_{i}}{\gamma_{i}+\mu_{i}} \int_{S} w_{t}(r) \bar{L}_{i t}(r) d r\right]^{\frac{1}{1+\varphi}},
$$

and, therefore using equation (19),

$$
\Pi_{t}=e_{t} E_{t}=e_{t}^{1+\varphi}=\sum_{i=1}^{I} \frac{\sigma_{i}}{\gamma_{i}+\mu_{i}} \int_{S} w_{t}(r) \bar{L}_{i t}(r) d r .
$$

Finally, competitive land and labor markets clear at each location, so equation (17) holds, and

$$
\bar{L}_{t}(r)=\sum_{i} \bar{L}_{i t}(r) .
$$


Dynamic competitive equilibrium. For a given period $t$ and a given distribution of fundamental amenities $\bar{a}(r)$, productivity $\tau_{i t}(r)$ and temperature $T_{t}(r)$, equations (2), (3), (4), (17), (19), (27), (28), and (31) pin down the world price of energy $e_{t}$, profits in the resource extraction sector $\Pi_{t}$, the distribution of population $\bar{L}_{t}(r)$, utility $u_{t}(r)$, amenities $a_{t}(r)$, land rents $R_{t}(r)$, and wages $w_{t}(r)$ across locations, as well as the distribution of price indices $P_{i t}(s)$ and sectoral employment $\bar{L}_{i t}(r)$ across sectors and locations. These conditions determine the period- $t$ equilibrium. Equation (13) gives the amount of innovation labor hired in each sector and each location. This, together with (9), yields the distribution of fundamental productivities in period $t+1, \tau_{i, t+1}(r)$. To update the distribution of temperature in $t+1, T_{t+1}(r)$, we use equations (20) to $(22)$.

\section{Quantification}

\subsection{Preliminaries}

From now onwards, we assume the economy has two sectors: agriculture $(A)$ and non-agriculture (which we denote by $M$, for manufacturing but includes all sectors that are not part of agriculture, including services). Further assume that we observe the matrix of bilateral trade costs $\varsigma(r, s)$, as well as land $H(r)$, temperature $T_{0}(r)$, total population $\bar{L}_{0}(r)$, the value of total output

$$
Y_{0}(r)=\frac{1}{\gamma_{A}+\mu_{A}} w_{0}(r) \bar{L}_{A 0}(r)+\frac{1}{\gamma_{M}+\mu_{M}} w_{0}(r) \bar{L}_{M 0}(r)
$$

and the value of agricultural output

$$
Y_{A 0}(r)=\frac{1}{\gamma_{A}+\mu_{A}} w_{0}(r) \bar{L}_{A 0}(r)
$$

at every location $r$ at time 0 . As we show in Appendix A, we can then use the model to recover the unique initial distributions of fundamental agricultural productivity, $\tau_{A 0}(r)$, fundamental non-agricultural productivity, $\tau_{M 0}(r)$, and fundamental amenities relative to utility, $\bar{a}(r) / u_{0}(r)$, that rationalize the data. We back out fundamental amenities $\bar{a}(r)$ by using subjective well-being data to measure $u_{0}(r)$, and we set moving costs $m_{2}(r)$ so that local changes in population between the first two periods coincide with what we observe in the data.

Appendix A also describes in detail the algorithm to compute an equilibrium of the model. The model can be solved forward, using only current data, for as many periods as needed.

\subsection{Data and Calibration}

We discretize the world into $64,8001^{\circ} \times 1^{\circ}$ cells. At that level of spatial resolution, our quantification requires initial distributions of population, $\bar{L}_{0}(r)$, total output, $Y_{0}(r)$, agricultural output, $Y_{A 0}(r)$, temperature, $T_{0}(r)$, as well as the distribution of land, $H(r)$. We also need estimates for bilateral transport costs, $\varsigma(r, s)$. Period 0 is taken to be the year 2000. Data on population, total output and land by grid-cell come from the G-Econ 4.0 database of Nordhaus et al. (2006). These data cover the entire globe, with the exception of a few countries: Afghanistan, Iraq, Libya, North Korea, Somalia, Turkmenistan and Zimbabwe. Estimates on bilateral transport costs come from Desmet et al. (2018). The data on agricultural output and temperature require some more explanation. 
Agricultural output and temperature. To estimate the initial distribution of agricultural output across grid-cells, we proceed in two steps. First, using high-resolution data on total crop production from GAEZ's Actual Yield and Production dataset, we compute grid-level agricultural production in year 2000 (IIASA and FAO, 2012). Second, we apply a country-specific conversion rate to local crop production so that its sum at the country level as a share of total output coincides with the share of value added that comes from agriculture, forestry, and fishing, as obtained from the World Bank Development Indicators. This is necessary because the GAEZ data do not include all agricultural activities. Figure 1 depicts the estimated distribution of agricultural output across the globe in year 2000. Agriculture is widespread across the globe. Regions with particularly high agricultural output include the U.S. Midwest, Europe, northern India, and eastern China.

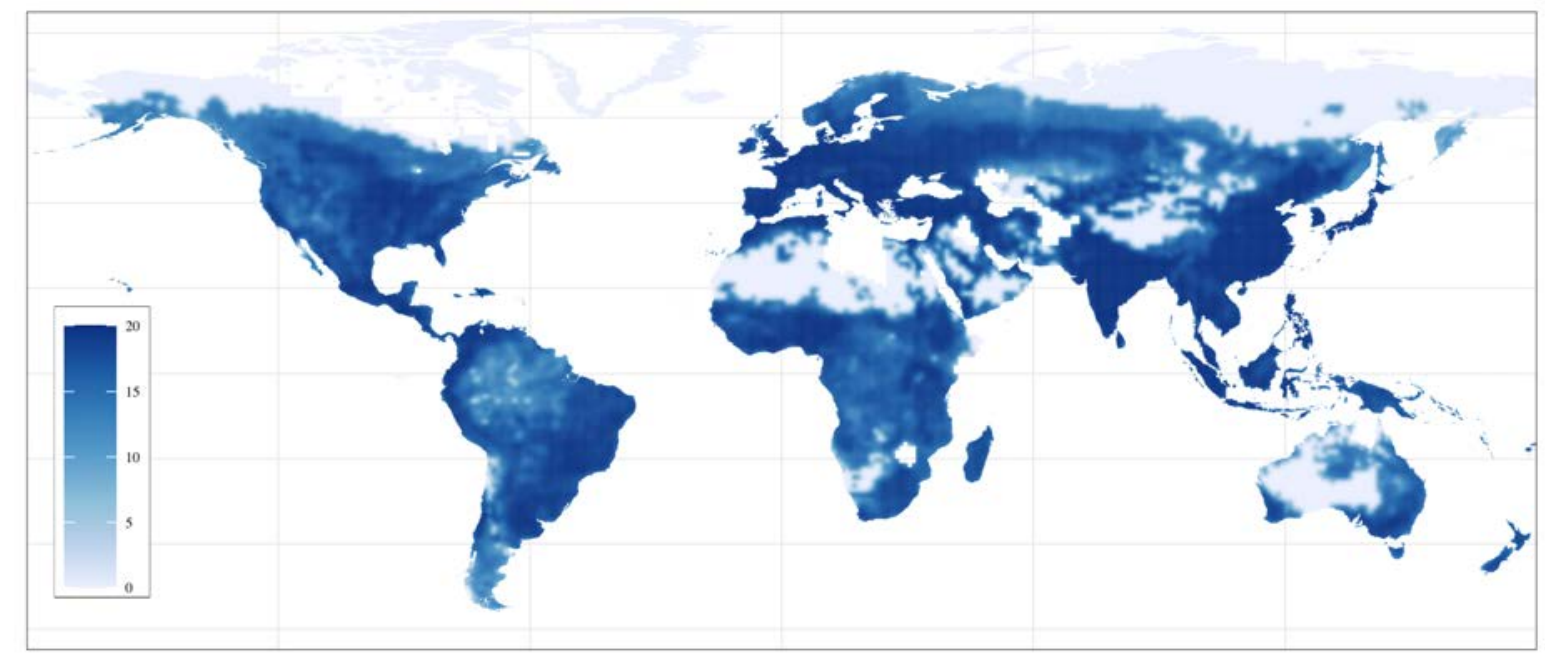

Note: Figure displays the log of agricultural output (US\$ million, PPP).

Figure 1: Agricultural Production in 2000

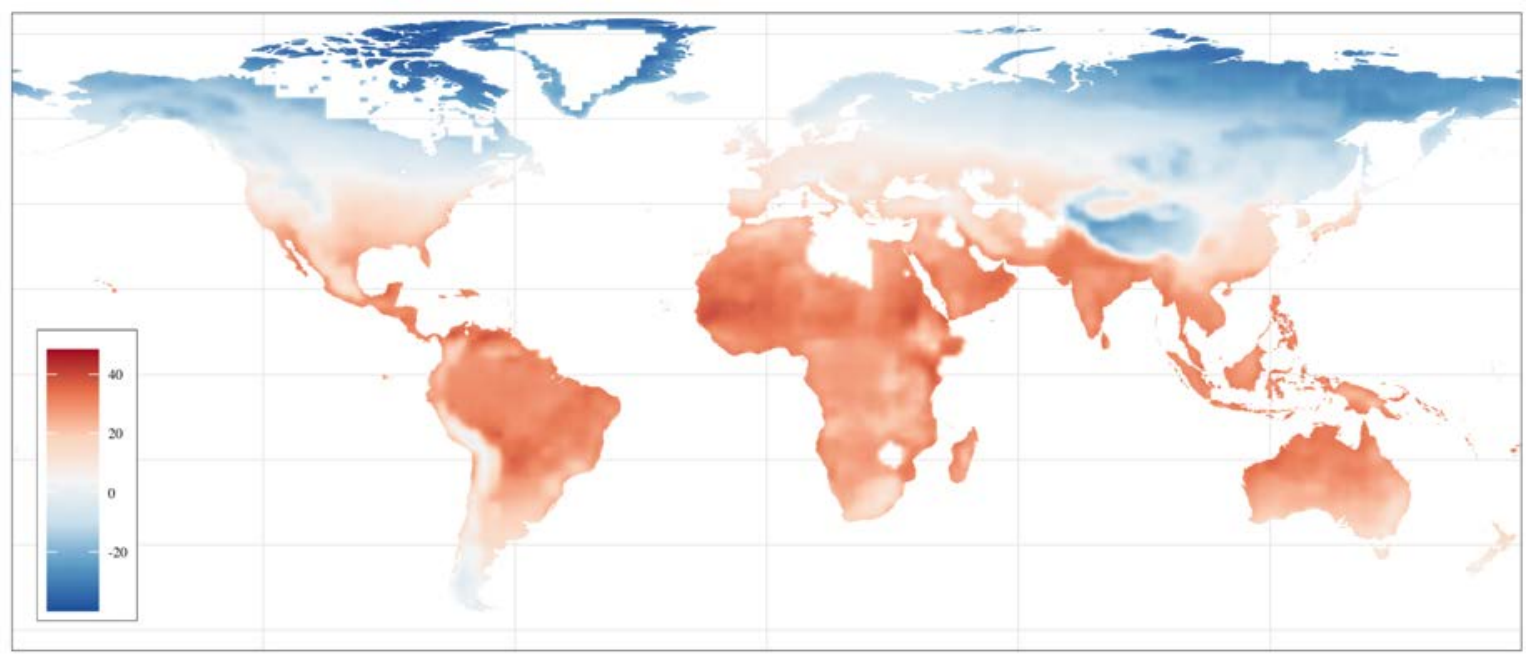

Figure 2: Average Temperature in $2000\left({ }^{\circ} \mathrm{C}\right)$ 
For the initial temperature distribution at the $1^{\circ} \times 1^{\circ}$ resolution we rely on data on yearly average temperature from the IPCC AR5 Data Distribution Center (IPCC, 2020). The resolution of the IPCC is $1.25^{\circ} \times 0.9^{\circ}$. When no centroid of any of these grid-cells lies within one of our $1^{\circ} \times 1^{\circ}$ grid-cells, we assign the temperature of the closest cell. Figure 2 depicts the world map of temperature in the year 2000. Much of the variation is across latitudes, but there are important exceptions: the Tibetan plateau, the Andes and the Rocky Mountains, for example, all have lower temperatures than their latitude would predict.

Table 1: Parameter Values

\begin{tabular}{|c|c|}
\hline Parameter & Target/Comment \\
\hline \multicolumn{2}{|l|}{ 1. Preferences } \\
\hline$\beta=0.96$ & Annual discount factor \\
\hline$\rho=0.75$ & Elasticity of substitution of $4^{1}$ \\
\hline$\lambda=0.32$ & Relation between amenities and population ${ }^{1}$ \\
\hline$\Omega=0.5$ & Elasticity of migration flows with respect to income ${ }^{1}$ \\
\hline$\psi=1.8$ & Subjective well-being parameter ${ }^{1}$ \\
\hline$\chi_{A}=0.051$ & Data on agricultural and total output \\
\hline$\chi_{M}=0.949$ & Data on agricultural and total output \\
\hline \multicolumn{2}{|l|}{ 2. Technology } \\
\hline$\alpha_{A}=0$ & No agglomeration externality in agriculture \\
\hline$\alpha_{M}=0.01$ & Agglomeration externality in non-agriculture ${ }^{1}$ \\
\hline$\theta=6.5$ & Trade elasticity ${ }^{1}$ \\
\hline$\mu_{A}=\mu_{M}=0.6$ & Labor share in agriculture and non-agriculture ${ }^{2}$ \\
\hline$\gamma_{A}=0.001$ & Growth rate of agricultural productivity ${ }^{3}$ \\
\hline$\gamma_{M}=0.0002$ & Growth rate of non-agricultural productivity ${ }^{3}$ \\
\hline$\sigma_{A}=0.04$ & Energy share in agriculture ${ }^{2}$ \\
\hline$\sigma_{M}=0.07$ & Energy share in non-agriculture ${ }^{2}$ \\
\hline$\delta=0.993$ & Technology diffusion $^{1}$ \\
\hline$\aleph=0.004$ & Spatial decay of diffusion ${ }^{4}$ \\
\hline$\phi=0.25$ & Energy supply elasticity ${ }^{2}$ \\
\hline \multicolumn{2}{|c|}{ 3. Temperature and carbon cycle } \\
\hline$g_{A}^{o p t}=19.9^{\circ} \mathrm{C}$ & Optimal temperature in agriculture ${ }^{2}$ \\
\hline$g_{A}^{v a r}=7.28^{\circ} \mathrm{C}$ & $0.1 \%$ of world agricultural production at locations below discount factor 0.01 \\
\hline$g_{M}^{o p t}=10.5^{\circ} \mathrm{C}$ & Relationship between non-agricultural productivity and temperature \\
\hline$g_{M}^{v a r}=11.0^{\circ} \mathrm{C}$ & Relationship between non-agricultural productivity and temperature \\
\hline$\varepsilon_{1}=0.9975$ & Decay of carbon stock ${ }^{2}$ \\
\hline$\varepsilon_{2}=0.29$ & 1200 GTC increase in global carbon stock by 2100 \\
\hline$\nu=0.0031$ & $3.7^{\circ} \mathrm{C}$ increase in global temperature by 2100 \\
\hline
\end{tabular}

Parameter values. Table 1 reports the parameter values we use. Many of them are taken from Desmet and Rossi-Hansberg (2015) and Desmet et al. (2018). Others are either calibrated to moments in the data or come from other papers. The innovation parameter in agriculture, $\gamma_{A}$, is set to match the growth rate in agricultural productivity between 1975 and 2000 in 30 countries as estimated by Duarte and Restuccia (2010). Similarly, the innovation parameter in non-agriculture, $\gamma_{M}$, is set to match the manufacturing productivity growth rate in the same countries over the same time period. The value for the spatial decay of technology diffusion, $\aleph$, falls within the range of values estimated for a set of different technologies by Comin et al. (2012). Consistent with the predictions for Representative Concentration Pathway (RCP) 8.5, we 
calibrate the carbon cycle parameter $\varepsilon_{2}$ to obtain a 1200 GTC increase in the stock of carbon by 2100 , and we set the parameter $\nu$ to obtain a $3.7^{\circ} \mathrm{C}$ global temperature increase by the end of the 21 st century. Before discussing the parameter values pertaining to the sensitivity of agricultural productivity and non-agricultural productivity to temperature, we need the initial distributions of temperature-adjusted productivity in both sectors, denoted by $\hat{\tau}_{i 0}(r)=\tau_{i 0}(r) g_{i}\left(T_{t}(r)\right), i \in\{A, M\}$.

Solving for initial distributions. Using the initial distributions of land, total population, total output and agricultural output, as well as estimates of trade costs and the parameter values in Table 1, we follow the procedure outlined in Appendix A to back out the distributions of the initial temperature-adjusted productivities in agriculture and non-agriculture, $\hat{\tau}_{i 0}(r)$. Determining the distributions of the initial fundamental productivities $\tau_{i 0}(r)$ will require estimates of the temperature discounts $g_{i}\left(T_{t}(r)\right)$, an issue we turn to below.

As outlined in Desmet et al. (2018), we then use data on subjective well-being from the Human Development Report to back out the distribution of fundamental amenities. Location-specific moving costs are then set so that the model-predicted changes in population between 2000 and 2005 match those in G-Econ $4.0 .^{8}$

Sensitivity of agriculture and non-agriculture to temperature. To disentangle fundamental productivity from temperature-adjusted productivity, we use the sector-specific temperature discount factor (10). Parametrizing this bell-shaped discount function requires for each sector $i$ estimates for the optimal temperature, $g_{i}^{\text {opt }}$, and for the variance of the relation between temperature and productivity, $g_{i}^{\text {var }}$.

For agriculture, Desmet and Rossi-Hansberg (2015) rely on agronomy studies to estimate an optimal growing-season temperature of $21.1^{\circ} \mathrm{C}$. Because we use annual average temperature, we need to map growingseason temperature into yearly average temperature. To that effect, we regress annual average temperature on growing-season temperature across all grid-cells, and use the estimated mapping to obtain an optimal annual average temperature in agriculture, $g_{A}^{o p t}$, of $19.9^{\circ} \mathrm{C}$. We then set the variance parameter of the agricultural temperature discount so that only $0.1 \%$ of world agricultural production takes place in locations with a discount factor below 0.01 . This yields $g_{A}^{v a r}=7.28^{\circ} \mathrm{C}$. The estimated agricultural temperature discount is depicted in Figure 3 Panel (a).

For non-agriculture, we take a different approach. We calibrate the parameter values of the temperature discount to the observed relation between temperature and the model-generated non-agricultural productivity across all grid-cells. ${ }^{9}$ To derive an estimating equation, we start by substituting the expression of the bell-shaped discount (10) into $\hat{\tau}_{M 0}(r)=\tau_{M 0}(r) g_{M}\left(T_{0}(r)\right)$. This yields a relation between the temperature-adjusted and the fundamental productivity in non-agriculture given by

$$
\log \hat{\tau}_{M 0}(r)=\log \tau_{M 0}(r)-\frac{1}{2}\left(\frac{T_{0}(r)-g_{M}^{o p t}}{g_{M}^{v a r}}\right)^{2} .
$$

\footnotetext{
${ }^{8}$ Agricultural output data indicates zero output in some cells of the world (particularly in deserts or polar regions). Given that our model and its inversion cannot handle zeros, we set the share of agricultural output in those regions to $10^{-12}$. In a few cases the reported agricultural output we obtain from GAEZ and the World Bank yields agricultural cell output levels that are larger than the total output level reported by G-Econ. In those cases we set the non-agricultural output share to $10^{-12}$.

${ }^{9}$ For non-agriculture, there is less guidance from the literature. For a discussion of the few studies that exist, see Dell et al. (2014).
} 
(a) Temperature discount

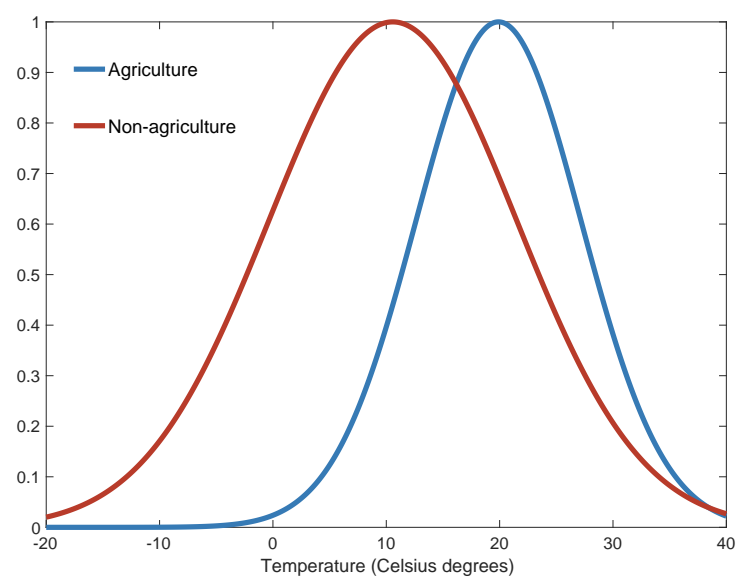

(b) Log non-agricultural productivity and temperature

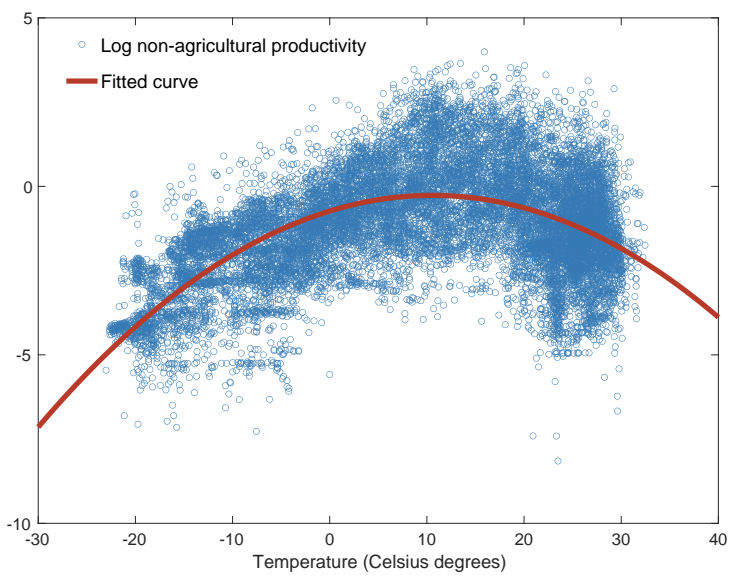

Figure 3: Temperature Discount in Agriculture and Non-Agriculture

Rearranging gives us the estimating equation

$$
\log \hat{\tau}_{M 0}(r)=\beta_{0}+\beta_{1} T_{0}(r)+\beta_{2} T_{0}(r)^{2}+\nu(r)
$$

where $\beta_{1}=g_{M}^{o p t} /\left(g_{M}^{v a r}\right)^{2}$ and $\beta_{2}=-1 /\left(2\left(g_{M}^{v a r}\right)^{2}\right)$. The identification assumption behind this estimation is that fundamental productivity $\tau_{M 0}(r)$ is uncorrelated with temperature. ${ }^{10}$ Using the model-generated values of $\hat{\tau}_{M 0}(r)$, we run regression (34). The underlying data of this regression, displayed in Figure 3 Panel (b), strongly suggest that the relation between the log of non-agricultural productivity and temperature is indeed quadratic, with the most productive locations corresponding to those with moderate temperatures. From the estimates of $\beta_{1}$ and $\beta_{2}$, we can derive the two parameters of the bell-shaped temperature discount factor on non-agricultural productivity, $g_{M}^{o p t}=10.5^{\circ} \mathrm{C}$ and $g_{M}^{v a r}=11^{\circ} \mathrm{C}$.

When comparing non-agriculture to agriculture, Figure 3 Panel (a) shows that its optimal temperature is lower and its sensitivity to temperature is smaller. These temperature discounts can be shown on a map. Panels (a) and (b) of Figure 4 depict the temperature discounts by location in the year 2000, $g_{A}\left(T_{0}(r)\right)$ and $g_{M}\left(T_{0}(r)\right)$. In agriculture relatively cold areas suffer the most: this explains the large discount on productivity in much of Canada and Russia. With the exception of some areas such as the Sahara desert, most regions closer to the Equator do not experience large productivity penalties. In non-agriculture, productivity does not suffer from much of a discount in large swaths of Canada and Russia, except in the northernmost areas. However, the lower optimal temperature in non-agriculture implies that warmer areas close to the Equator experience large productivity penalties.

Our estimates of the temperature discounts allow us to back out the initial distributions of fundamental productivity in agriculture and non-agriculture, displayed in Panels (c) and (d) of Figure 4. We see that large areas of China, Central Asia, and Canada have relatively high agricultural productivity, in spite of the important temperature discount they suffer. As global warming reduces the temperature penalty in

\footnotetext{
${ }^{10}$ This is plausible if temperature has had no effect on past investment decisions. Of course, if temperature has been an important determinant of the areas where humanity has concentrated and flourished, then the coefficients $\beta_{1}$ and $\beta_{2}$ are likely to include not just the direct effect of temperature on non-agricultural productivity, but also its indirect effect through cumulative past innovation. If so, we would be overstating the effect of temperature on non-agricultural productivity, because in our theory it is meant to only capture its direct effect.
} 
northern latitudes, these regions stand to become some of the more productive in agriculture. Areas of high non-agricultural productivity tend to be spatially concentrated in the developed world, such as North America, western Europe, Japan, and southeastern Australia. Some areas in Brazil, Paraguay and Argentina, as well as parts of Central Africa, display markedly lower productivity in non-agriculture than neighboring regions. These are areas that heavily specialize in agriculture in the data, and so have limited experience in other sectors.

(a) Temperature discount in agriculture

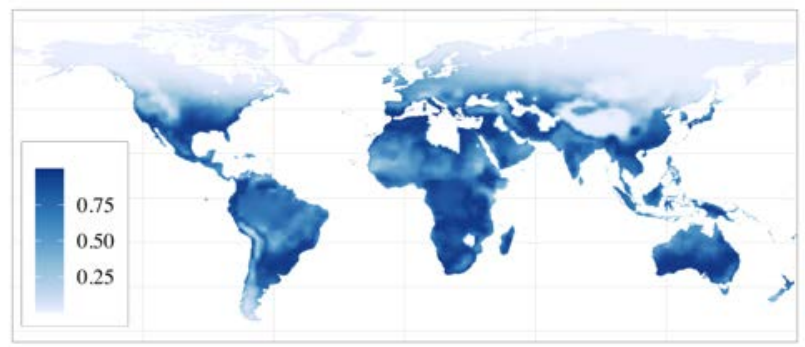

(c) Fundamental productivity in agriculture

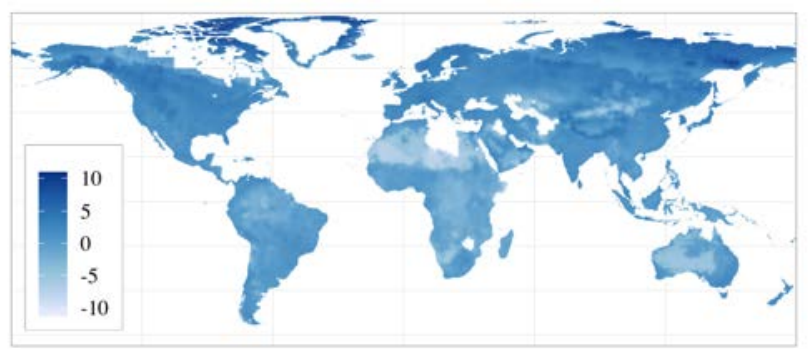

(b) Temperature discount in non-agriculture

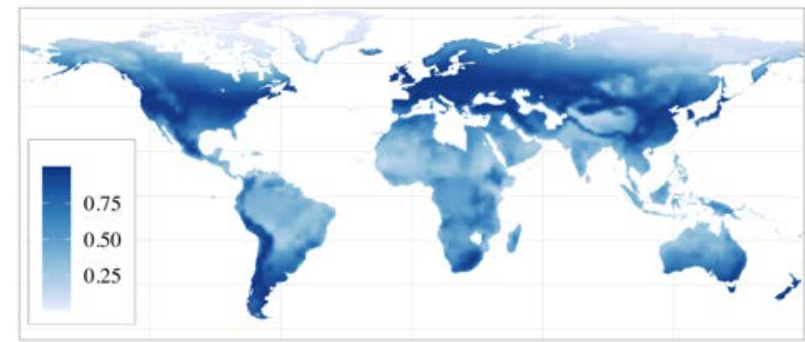

(d) Fundamental productivity in non-agriculture

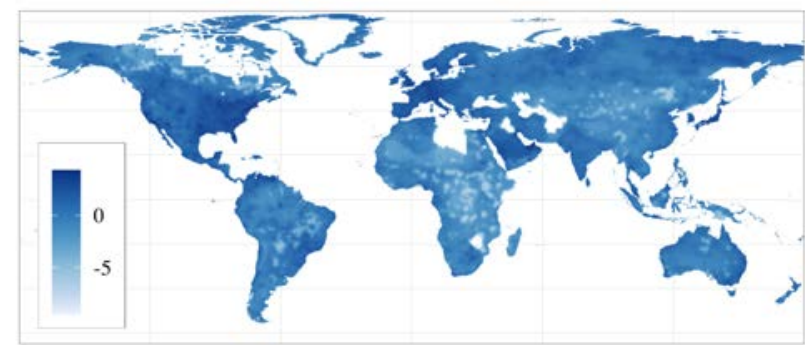

Note: Panel (a) depicts $g_{A}\left(T_{0}(r)\right)$, Panel (b) depicts $g_{M}\left(T_{0}(r)\right)$, Panel (c) depicts $\log \left(\tau_{A 0}(r)\right)$, and Panel $(\mathrm{d}) \operatorname{depicts} \log \left(\tau_{M 0}(r)\right)$.

Figure 4: Temperature Discount and Fundamental Productivity in 2000

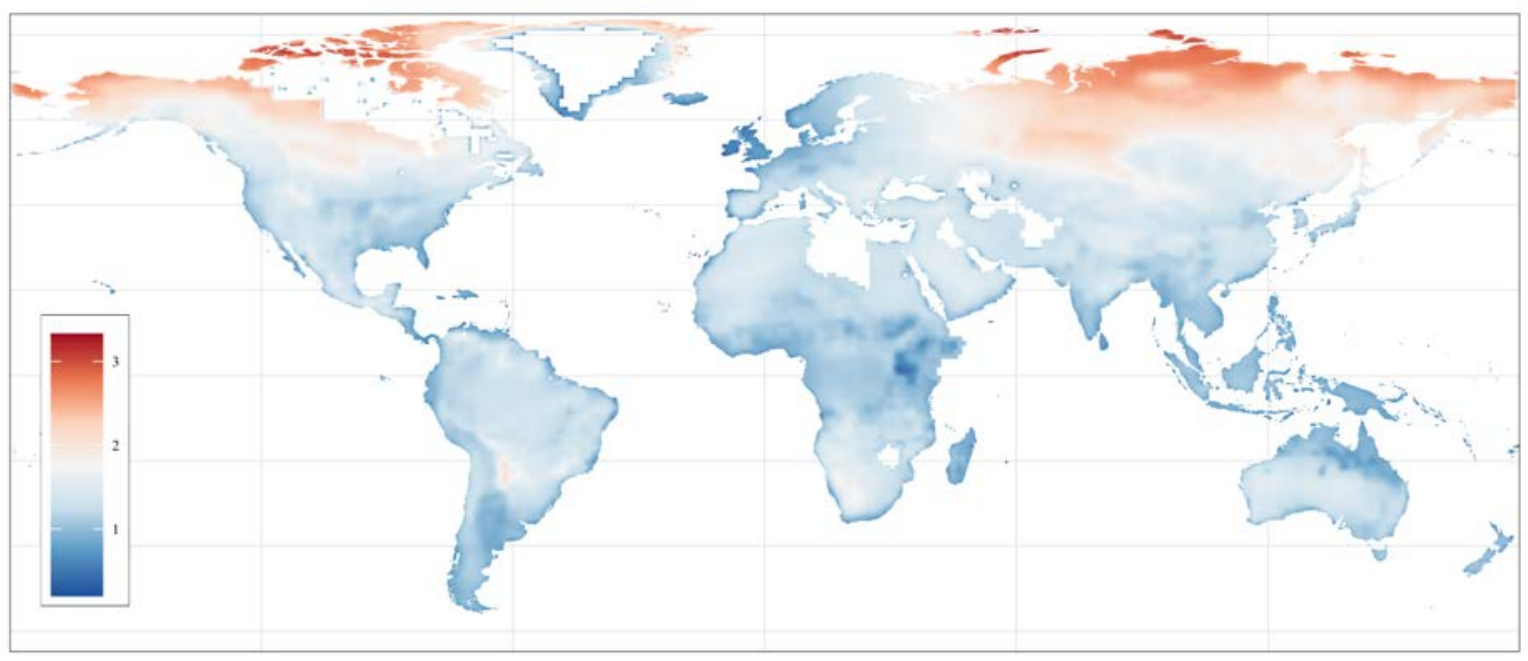

Figure 5: Predicted Change in Temperature for $1^{\circ} \mathrm{C}$ Increase in Global Temperature 
Model simulation. To simulate the model forward, we use the equilibrium allocation in period $t$ to estimate fundamental productivities in period $t+1$, and we use the carbon cycle, as well as the relation between the change in the carbon stock and the change in temperature in (22), to get estimates of temperature by location.

An important feature of global warming is that the rise in temperature is predicted to be heterogeneous across space. Polar latitudes are expected to experience higher-than-average increases in temperature, whereas coastal regions, including some islands such as Britain, are set to experience lower-than-average increases in temperature. To get estimates of the location-specific parameter $\xi(r)$ that measures the local increase in temperature for a one-degree global increase in temperature, we use twenty-year intervals of predicted local and global temperatures between 2000 and 2100 from the IPCC AR5 Data Distribution Center (IPCC, 2020) for RCP 8.5, and run the regression

$$
T_{t}(r)-T_{t-1}(r)=\xi(r)\left(T_{t}-T_{t-1}\right)+v_{t}(r)
$$

Figure 5 depicts the spatial distribution of $\xi(r)$. As can be seen, in some polar areas a one-degree increase in global temperature is predicted to translate into a more than three-degree increase in local temperatures.

With the distributions of fundamental productivities and temperatures in $t+1$, we then solve for sectoral employment levels, wages and prices in $t+1$. Using this algorithm we can compute the equilibrium allocation for as many periods as necessary.

\section{Global Warming and Local Specialization}

We now simulate our model forward for 200 years and analyze how global warming affects the world's economic geography. The effect of rising temperatures is location- and sector-specific. As a result, residents of particularly hard-hit locations may have an incentive to relocate, and local sectoral specialization may shift because of changing comparative advantage. Our goal is to document the spatial response of population, income per capita and specialization to global warming. We are also interested in the aggregate effects of climate change. Needless to say, the magnitude of spatial frictions affects the extent of these adjustments. In our baseline simulation we keep mobility frictions at their current levels. Given our interest in trade and specialization, we then explore the effects of higher or lower trade costs.

\subsection{Current Spatial Frictions}

Spatial distribution of population and output per capita. Figure 6 depicts the log difference of population in 2200 between the baseline with climate change and a counterfactual exercise with constant temperatures. Although the impact of climate change on population across the world is large and heterogeneous, with some regions doubling and others reducing by half their 2200 population, it does not dramatically affect the geography of the world's main population centers. ${ }^{11}$ Many of today's densest regions, such as western Europe, India, and eastern China, continue to be densely populated two centuries from now, whether the world experiences climate change or not. There are some significant climate-induced shifts though. Northern latitudes gain in population density, at the expense of regions such as the Arabian peninsula, northern India, western Australia, northern Africa, Brazil and Central America. In the regions that lose population, inland

\footnotetext{
${ }^{11}$ Because the variation in population density in the world in 2000 and 2200 is as large as $15 \log$ points, changes by 1 log point do not drastically alter the density map.
} 
areas tend to be more impacted by rising temperatures than coastal areas. This could reflect temperatures rising less in regions close to oceans; it could also reflect the greater resilience of coastal agglomerations due to their better connectivity to the rest of the world.

In terms of real output per capita, Figure 7 shows that over the next two centuries the more northern latitudes of Canada and Siberia improve their lot because of rising temperatures, whereas sub-Saharan Africa becomes worse off. The losses from climate change are more widespread in the case of real output per capita than in the case of population: almost all regions spanning the latitudes comprised between southern Africa to the south and the Mediterranean basin to the north lose output per capita. These more widespread losses are related to two factors. First, whereas the relocation of population is a zero-sum game, this is not the case with global output per capita. Second, the relocation of population is limited by mobility restrictions, implying that certain areas that suffer significant negative shocks to output per capita may lose relatively fewer people.

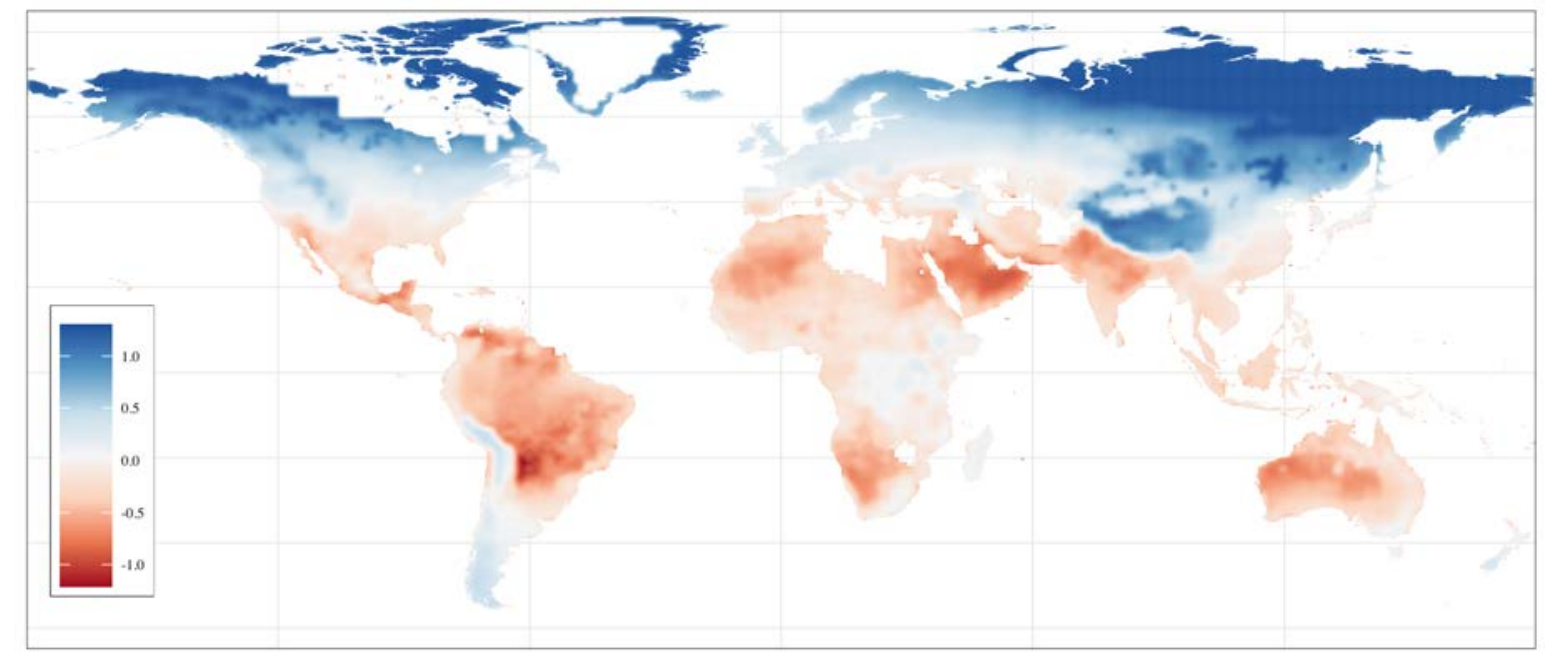

Note: Figure displays $\log \left(\bar{L}_{200}(r)\right)$ under climate change minus $\log \left(\bar{L}_{200}(r)\right)$ under no climate change.

Figure 6: Effect of Climate Change on Predicted Population in 2200

Sectoral specialization. What is the role of agriculture and non-agriculture in these geographic shifts? When considering agricultural output, Panels (a), (b) and (c) of Figure 8 show that over the next two centuries agriculture is predicted to experience increased geographic concentration. Climate change has a pronounced effect on where agriculture continues to be prominent. In the absence of rising temperatures, South America and sub-Saharan Africa maintain their importance, in addition to India, eastern China and eastern Europe. Today's developed world is predicted to specialize almost fully in non-agricultural sectors. With climate change, Canada emerges as a major agricultural producer, as do Russia and Central Asia. This comes at the expense of declining production in India, South America, and sub-Saharan Africa. The regions that gain from climate change are relatively cold areas with high fundamental productivity. Global warming reduces the temperature penalty they suffer, making them highly productive.

With non-agriculture being less sensitive to rising temperatures than agriculture, we might have expected equatorial regions to become non-agricultural powerhouses. However, Figure 9 shows no evidence of this happening. Two reasons explain this. First, the optimal temperature for non-agriculture is lower than for agriculture, implying that any increase in temperature in equatorial regions leads to a loss in non- 
agricultural productivity. Second, their original productivity in non-agriculture is relatively low, and they are unable to catch up with more advanced economies.

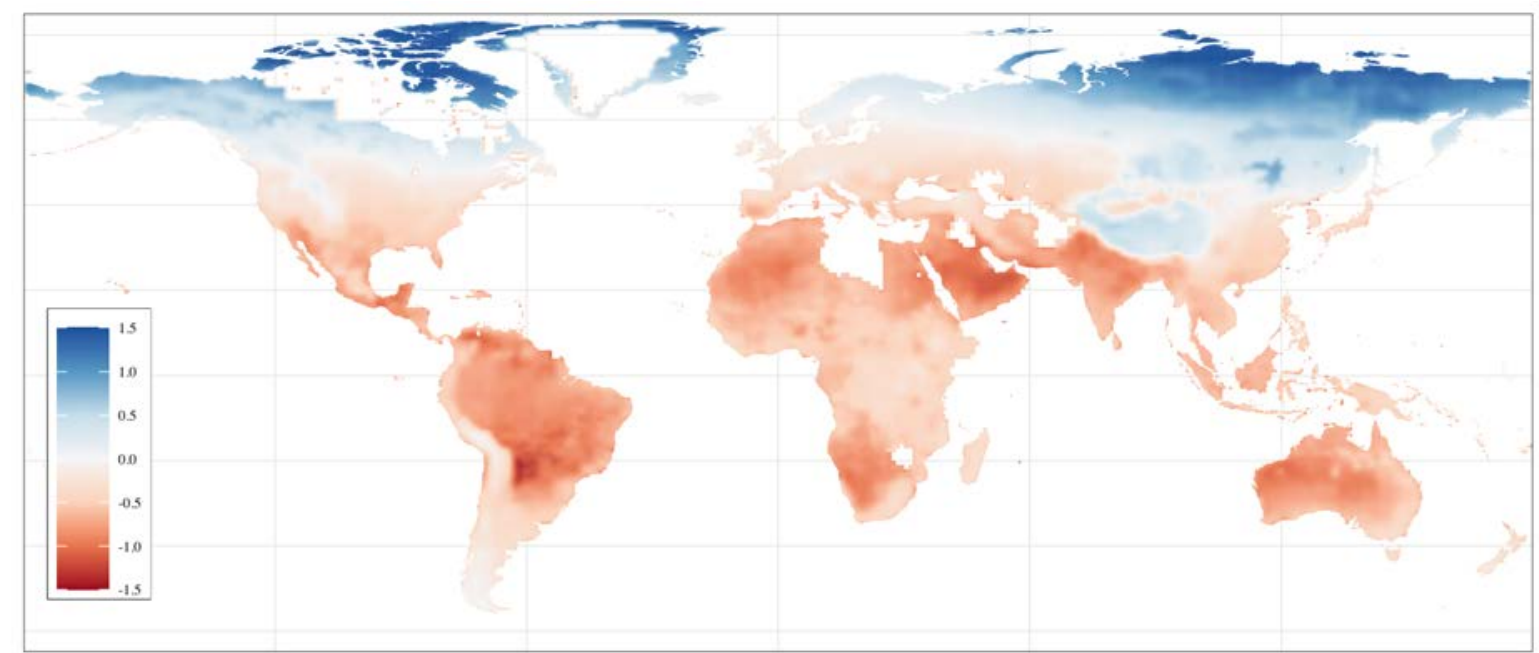

Note: Figure displays the log of real output per capita under climate change minus the log of real output per capita under no climate change in period 200.

Figure 7: Effect of Climate Change on Real Output per Capita in 2200

(a) Agricultural output in 2000

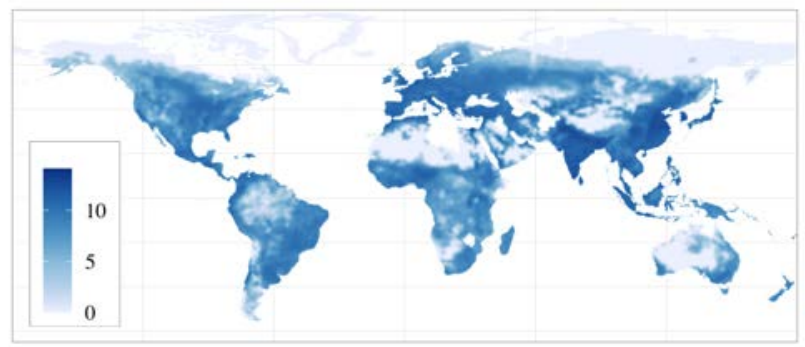

(c) Agricultural output in 2200: climate change

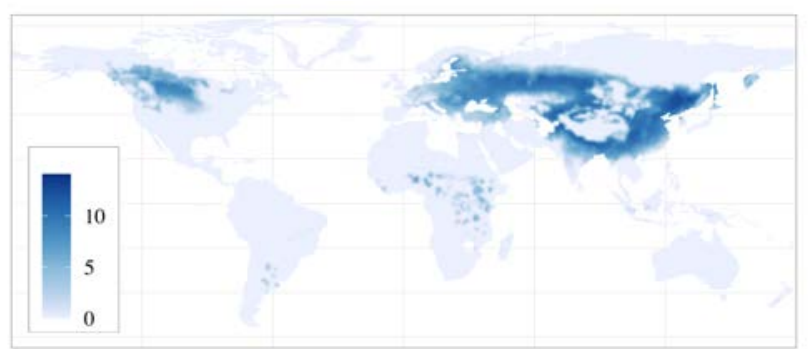

(b) Agricultural output in 2200: no climate change

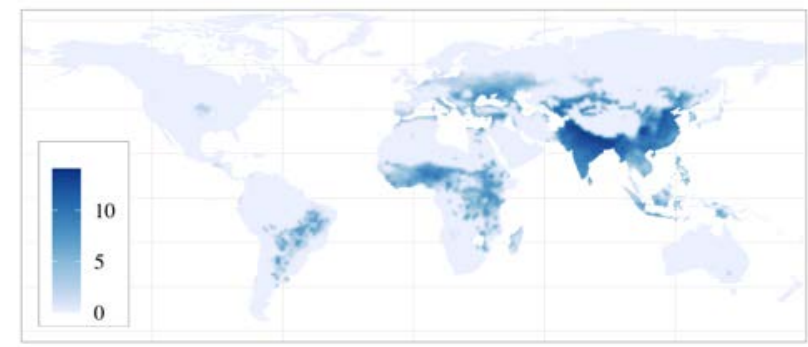

(d) Ag. output in 2200: climate - no climate change

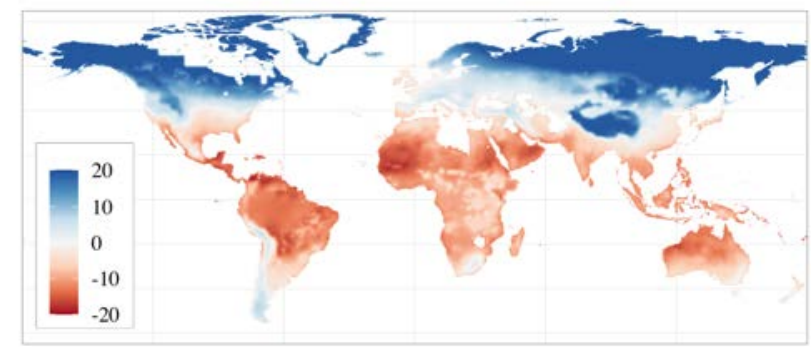

Note: Panel (a) displays the log of agricultural output in period 1, Panel (b) displays the log of agricultural output in period 200 under no climate change, Panel (c) displays the log of agricultural output in period 200 under climate change, and Panel (d) displays the log difference of agricultural output in period 200 with and without climate change. In all panels agricultural output is normalized by average nominal wages in the world.

Figure 8: Agricultural Output 
(a) Non-Agricultural output in 2000

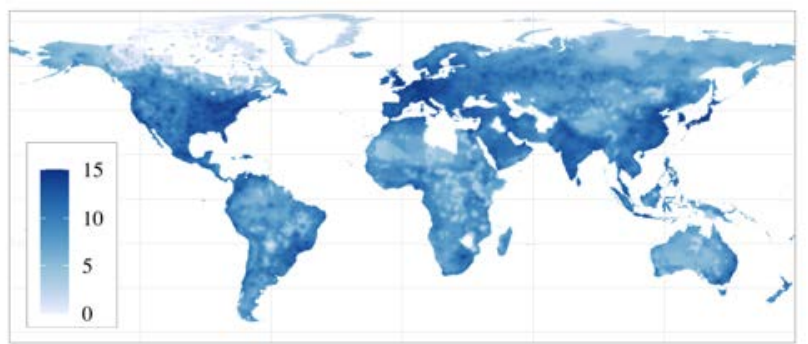

(c) Non-Agricultural output in 2200: climate change

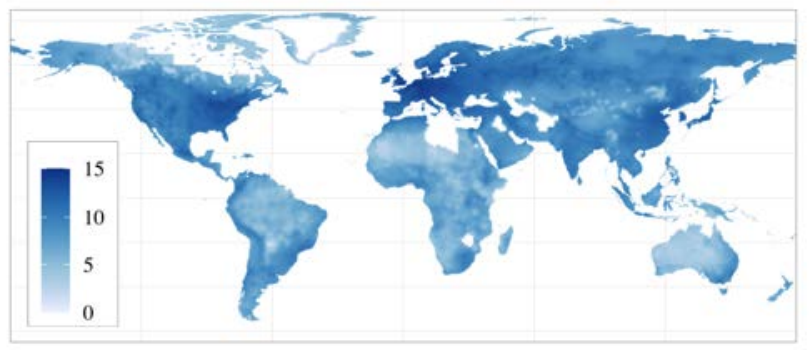

(b) Non-Agricultural output in 2200: no climate change

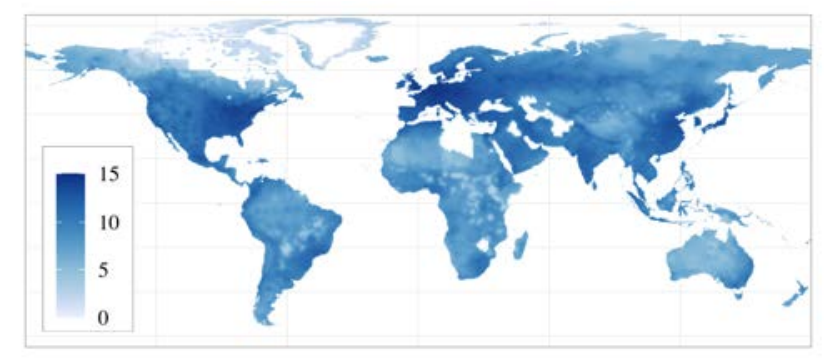

(d) Non-Ag. output in 2200: climate - no climate change

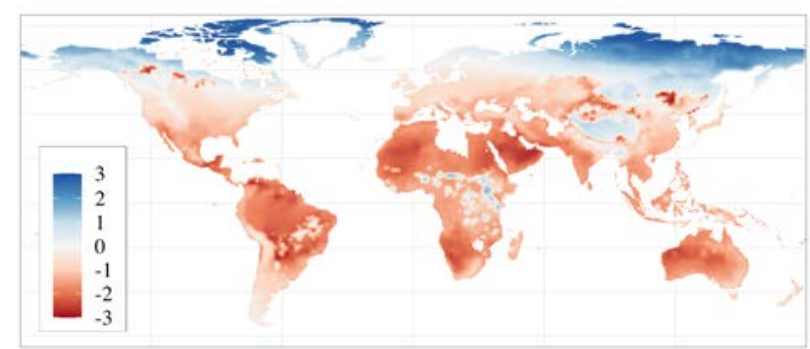

Note: Panel (a) displays the log of non-agricultural output in period 1, Panel (b) displays the log of non-agricultural output in period 200 under no climate change, Panel (c) displays the log of non-agricultural output in period 200 under climate change, and Panel (d) displays the log difference of non-agricultural output in period 200 with and without climate change. In all panels agricultural output is normalized by average nominal wages in the world.

Figure 9: Non-Agricultural Output

When focusing on specialization patterns in terms of sectoral employment shares, Panels (a), (b) and (c) of Figure 10 confirm that the areas that specialize in agriculture become much more concentrated in space. While in the year 2000 we still have many locations with agricultural employment shares above $20 \%$ scattered around the globe, by the year 2200 areas of high agricultural specialization are limited to a few regions. In the absence of climate change, those regions are concentrated in South America, sub-Saharan Africa and India's Ganges Valley. With rising temperature, clusters of agricultural specialization shift to Central Asia, China and Canada.

Aggregate patterns. At the aggregate level, Panel (a) of Figure 11 shows that the growth rate of world real GDP per capita is predicted to increase from $2.2 \%$ annually in 2000 to $2.8 \%$ annually in 2200 , further increasing to $3.0 \%$ by the year $2400 .{ }^{12}$ Global warming leads to a loss in the level of world real GDP per capita of around $6 \%$ by the year 2200 , increasing to around $9 \%$ by the year 2400 (Panel (b)). In terms of world utility, Panel (a) shows that its growth rate rises from $2.1 \%$ in 2000 to $2.7 \%$ in 2200 and $2.9 \%$ in 2400 . The losses due to global warming are greater than in the case of real GDP per capita: more than $15 \%$ by 2200 and above $20 \%$ by 2400 (Panel (b)). To understand why losses from global warming are larger for utility than for real income per capita, recall that utility takes into account amenities whereas real GDP per capita does not. As global warming tends to benefit locations at more polar latitudes which on average have worse amenities, rising temperatures have a more negative effect on utility than on income per capita. ${ }^{13}$

\footnotetext{
${ }^{12}$ To sharpen the visualization of some of the very long-run trends, it is convenient to simulate the model for another 200 years, until the year 2400 .

${ }^{13}$ This effect would be mitigated if we were to allow for amenities to change with temperature as in Cruz and Rossi-Hansberg (2020).
} 
(a) Agricultural specialization in 2000

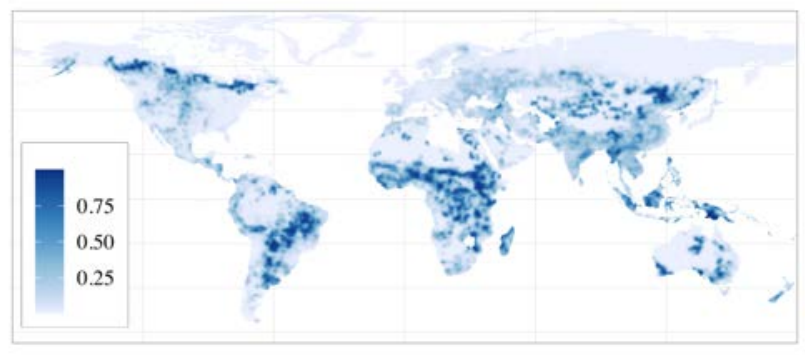

(c) Ag. specialization in 2200: climate change

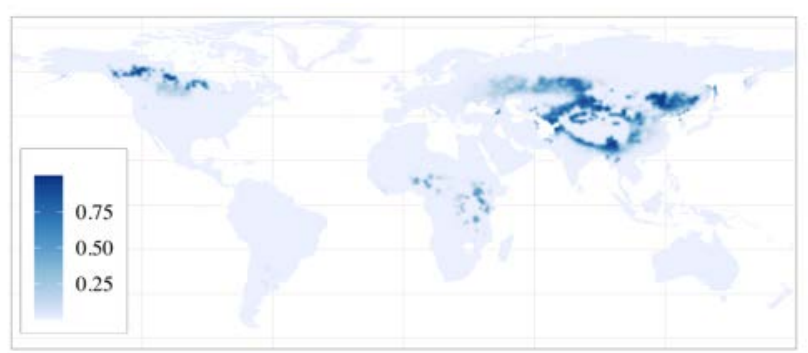

(b) Ag. specialization in 2200: no climate change

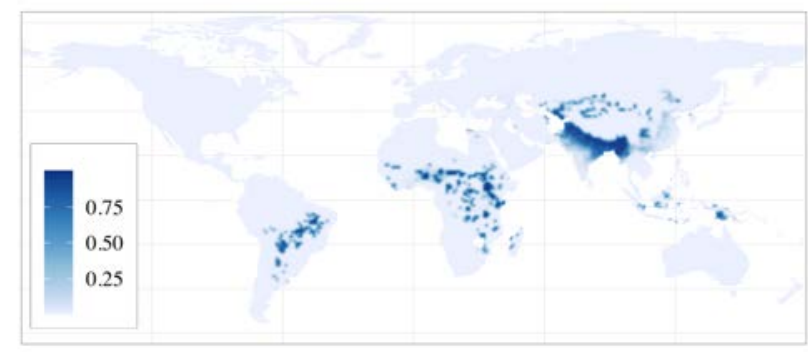

(d) Ag. spec. in 2200: climate - no climate change

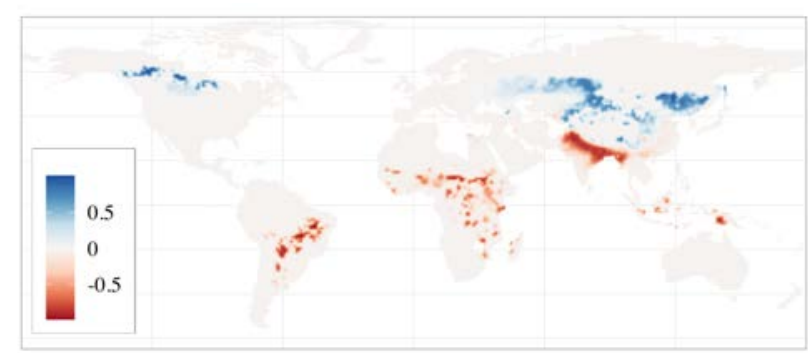

Note: Panel (a) displays share of labor employed in agriculture in period 1, Panel (b) displays the share of labor employed in agriculture in period 200 under no climate change, Panel (c) displays the share of labor employed in agriculture in period 200 under climate change, and Panel (d) displays the difference in the share of labor employed in agriculture with and without climate change in period 200 .

Figure 10: Agricultural Specialization

(a) Growth rates with climate change

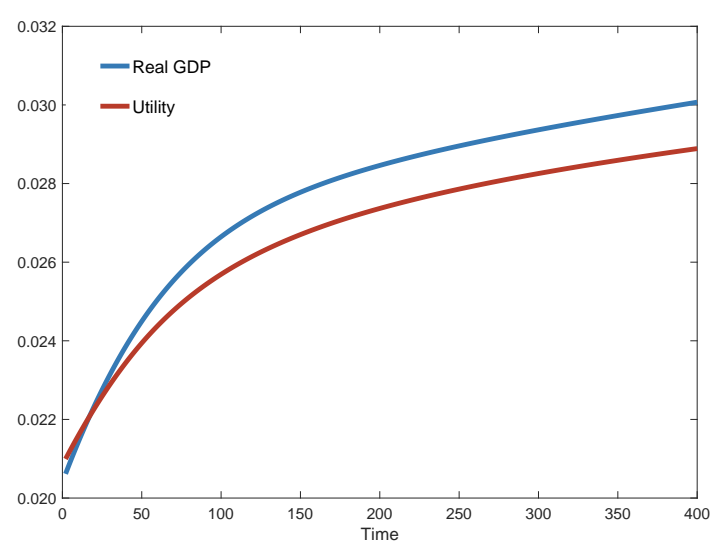

(b) Log difference: climate - no climate change

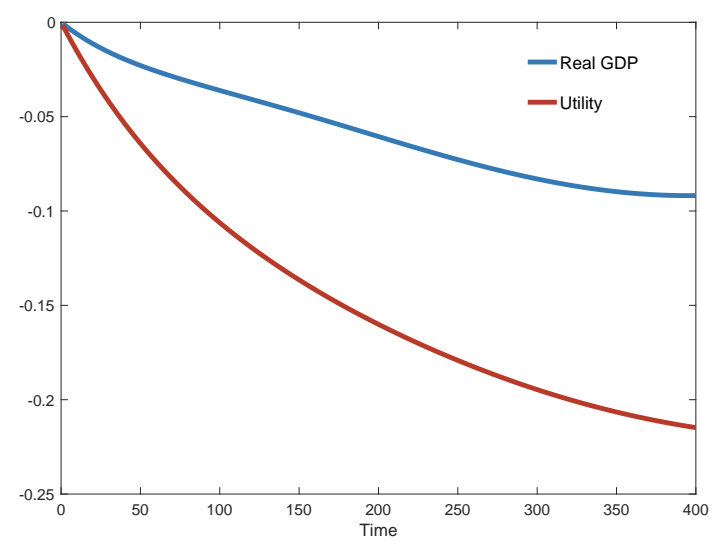

Figure 11: Aggregate Real GDP per Capita and Utility: Growth Rates and Levels

Two other aggregate effects from global warming are worth mentioning. First, Figure 12 Panel (a) shows that higher global temperatures lower the growth rate of non-agricultural productivity, and increase the growth rate of agricultural productivity. Given that agriculture is more sensitive to rising temperatures than non-agriculture, we might have expected the contrary. However, global warming shifts agriculture to areas with relatively high exogenous productivity. As an example, in a world without climate change, one of the regions with the highest agricultural employment share is northern India. Global warming shifts agricultural 
(a) Productivity growth: climate - no climate change

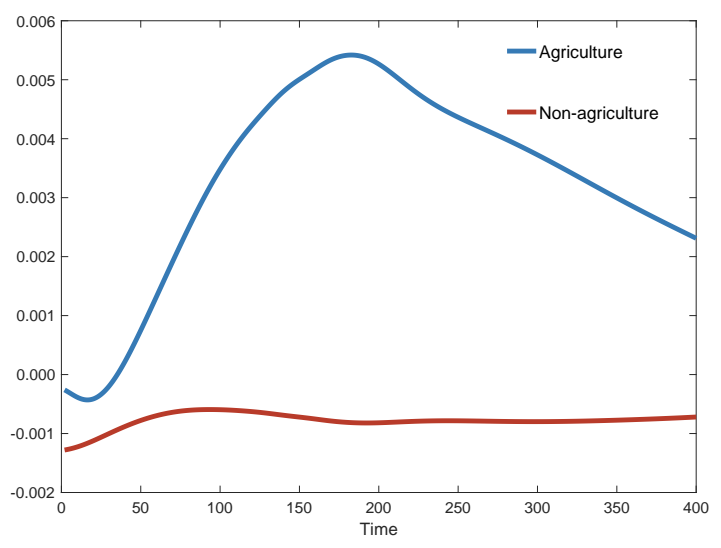

(b) Agricultural employment (billions)

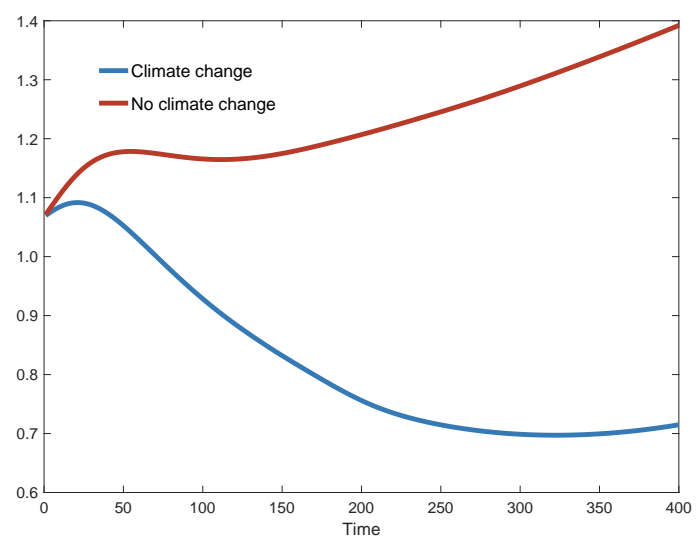

Figure 12: Productivity Growth and Employment

specialization to parts of Central Asia and Canada, which have higher fundamental agricultural productivity. Second, Figure 12 Panel (b) shows that climate change leads to lower agricultural employment. Given that our preferences are Cobb-Douglas in agriculture and non-agriculture, this is not a simple counterpart of the relative increase in agricultural productivity under climate change. Instead, it has to do with global warming pushing agriculture to regions where labor is relatively expensive. More specifically, Figure 8 shows that, with climate change, agricultural production moves to areas where land is abundant relative to labor (e.g. northern Canada, Russia, and Mongolia), so total agricultural employment falls. In contrast, without climate change, agriculture concentrates more in India, where labor is relatively abundant, so total agricultural employment increases.

\subsection{Trade Costs}

We now explore how different levels of trade costs affect the world's economic geography. In particular, we compare a world with $50 \%$ higher trade costs to one with $50 \%$ lower trade costs.

Spatial distribution of population and GDP per capita. Consider first the effect of trade costs on the spatial distribution of population in the year 2200 in a world with climate change. Figure 13 depicts the log difference in population when we increase trade costs by $50 \%$ relative to a scenario where we decrease them by $50 \%$. As the figure shows, in a world with high transport costs, population is more concentrated in today's developed countries and regions, such as the U.S., Europe, and Japan. Living far from the main production centers becomes more costly, so population concentrates. This also explains why Africa, South America, and Australia all lose considerable fractions of their population in the high trade cost scenario.

Now consider the effect that trade costs have on the impact of climate change across the world. Figure 14 presents a difference-in-difference visualization of this effect. More specifically, it displays the difference between high and low trade costs in the climate-induced log difference in population in the year 2200. The figure shows that with high trade costs more people leave the areas that are estimated to suffer the most from global warming. That is, with higher trade costs we witness more relocation of people from regions closer to the Equator to areas closer to the poles. This suggests that trade and migration are substitutes. When faced with a climate-induced sectoral shock, higher trade costs limit the scope of locally adjusting by 
changing specialization. That makes adjusting through migrating relatively more attractive. The differencein-difference graph for GDP per capita exhibits a very similar pattern, with production moving out of South America, Africa, and South Asia when trade costs are high, and concentrating in Canada and Siberia. We omit the map for brevity.

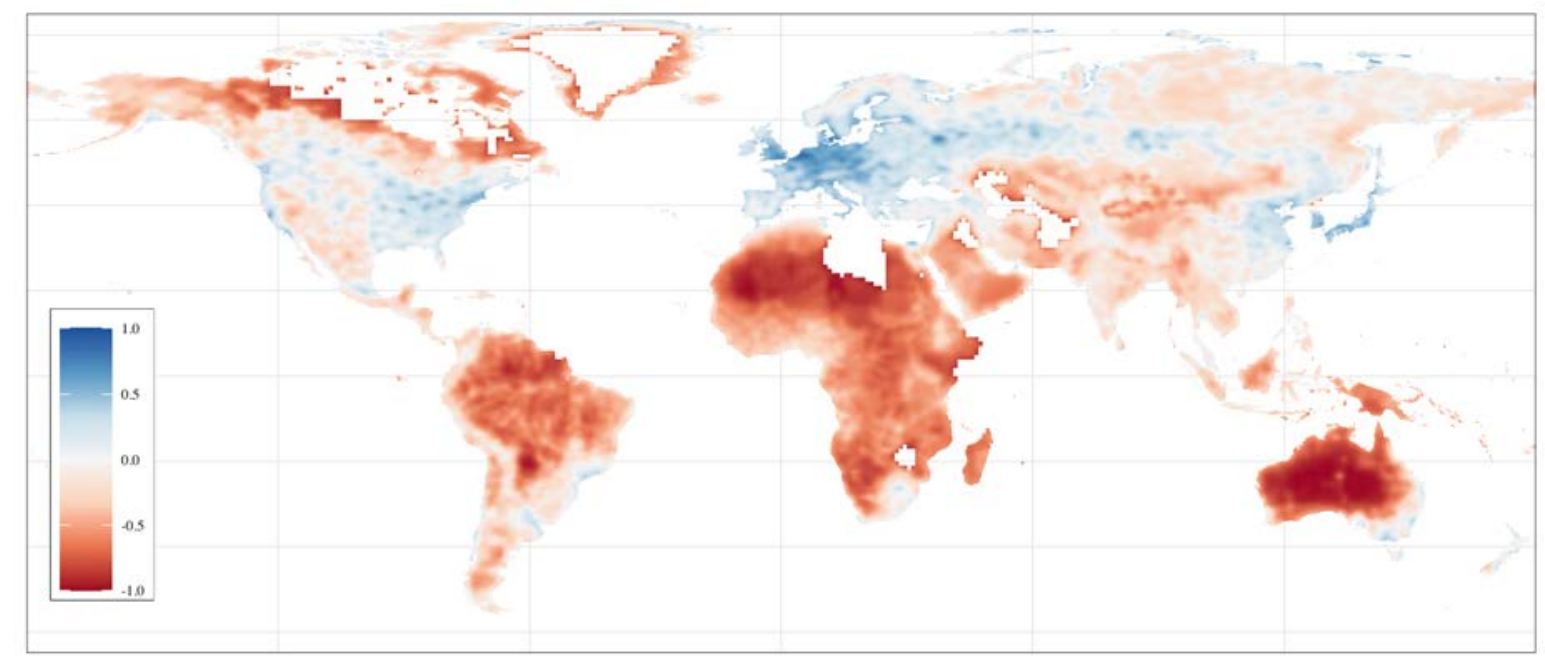

Note: Figure displays the difference in population with climate change in period $200, \log \left(\bar{L}_{200}(r)\right)$, with high $(+50 \%)$ minus with low $(-50 \%)$ trade costs.

Figure 13: The Effect of Trade Costs on Population in 2200

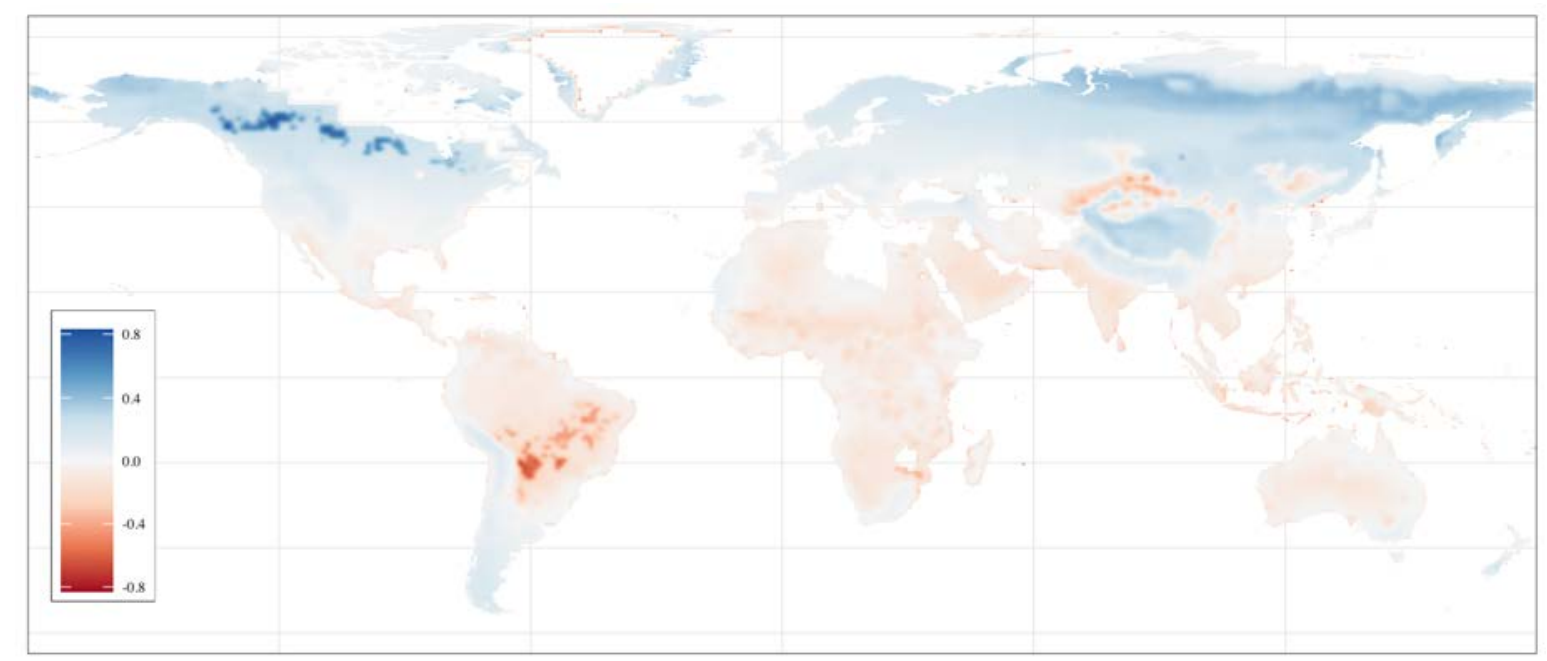

Note: Figure displays the difference with high $(+50 \%)$ minus low $(-50 \%)$ trade costs of the difference in $\log \left(\bar{L}_{200}(r)\right)$ with minus without climate change

Figure 14: The Impact of Trade Costs on the Effect of Climate Change on Population in 2200

Sectoral specialization. Panels (a) and (b) of Figure 15 show that the predicted distribution of agricultural production in the year 2200 is more dispersed under high trade costs than under low trade costs, for the simple reason that people source goods from closer by. In particular, with high trade costs, Europe, 
North America, as well as parts of South America and sub-Saharan Africa, continue to be important agricultural producers. In addition, agriculture stretches into more northern latitudes of Siberia. When comparing climate change to no climate change in Panels (c) and (d), under low trade costs we see a resurgence of agriculture at moderate latitudes in Europe, Russia and North America. This resurgence is not present when trade costs are higher, because in that case these regions continue to be agricultural producers even in the absence of climate change.

When looking at the employment share in agriculture in the year 2200, Panels (a) and (b) of Figure 16 similarly display greater geographic dispersion under high trade costs. Rising temperatures lead to relocations that are also more spatially dispersed when trade costs are high. More specifically, in a world with high trade costs, agriculture relocates to many areas of East and Central Asia, as well as to more northern latitudes in Canada, whereas in a world with low trade costs, relocation occurs most prominently from northern India to parts of China and Central Asia. Note that the climate-induced resurgence of Europe and the US as agricultural producers if trade costs are low (Figure 15 Panel (d)) does not translate in a substantial increase in agricultural employment (Figure 16 Panel (d)), suggesting that these regions benefit from high-productivity agriculture that requires little labor.

(a) Agricultural Output: high trade costs

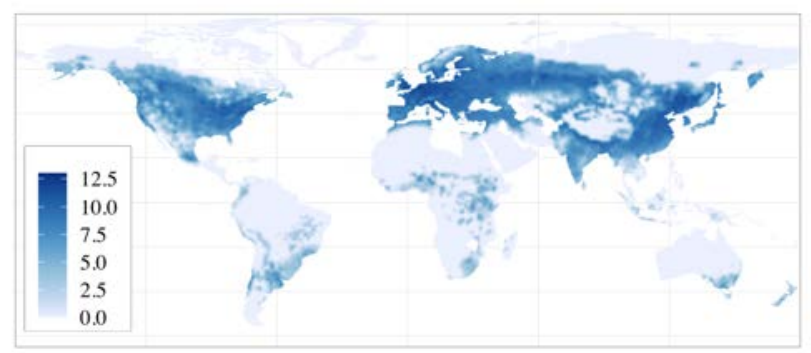

(c) Effect of climate on ag. output: high trade costs

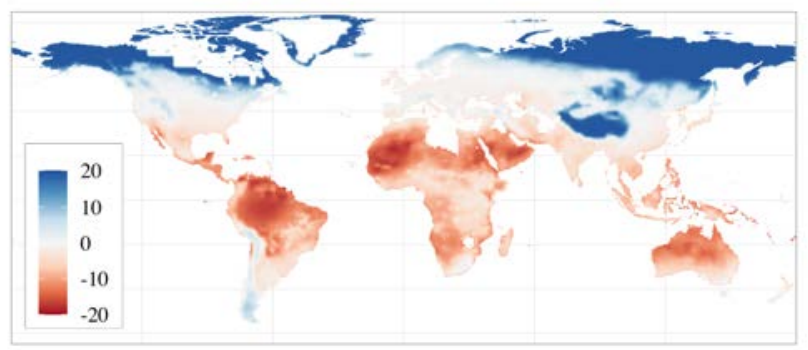

(b) Agricultural Output: low trade costs

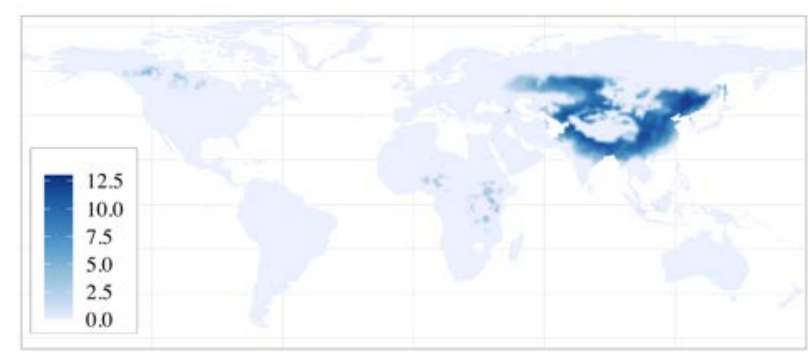

(d) Effect of climate on ag. output: low trade costs

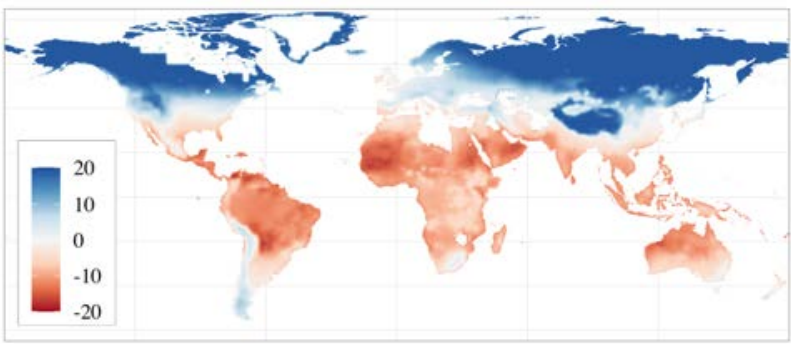

Note: Panel (a) displays log of agricultural output under climate change with $+50 \%$ trade costs, Panel (b) displays log of agricultural output under climate change with $-50 \%$ trade costs, Panel (c) displays log difference of agricultural output with and without climate change $(+50 \%$ trade costs), Panel (d) displays log difference in agricultural output with and without climate change (-50\% trade costs). In all panels agricultural output is normalized by average nominal wages in the world. All maps are for period 200.

Figure 15: Agricultural Output and Trade Costs

When looking at non-agricultural output in Figure 17, higher temperatures lead to relocations to higher latitudes. There are some exceptions though: under high trade costs, we see some areas in northern Canada losing non-agriculture, and a number of dispersed regions in Africa as well as the Tibetan Plateau gaining non-agriculture. These areas tend to be mirror images of the shifting specialization patterns observed in Figure 16 Panel (a). For example, northern Canada switches specialization, from non-agriculture 
(a) Ag. specialization: high trade costs

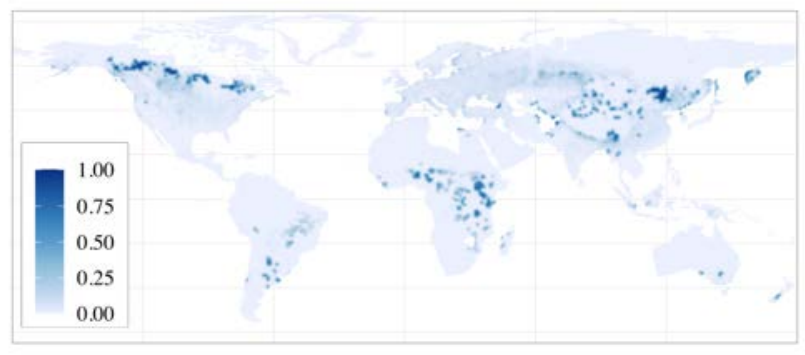

(c) Effect of climate on ag. spec.: high trade costs

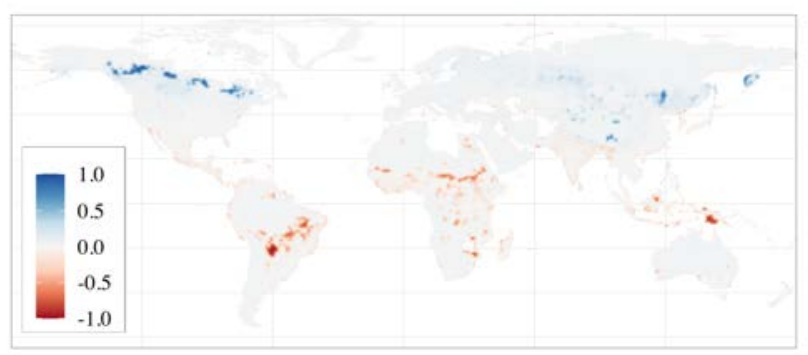

(b) Ag. specialization: low trade costs

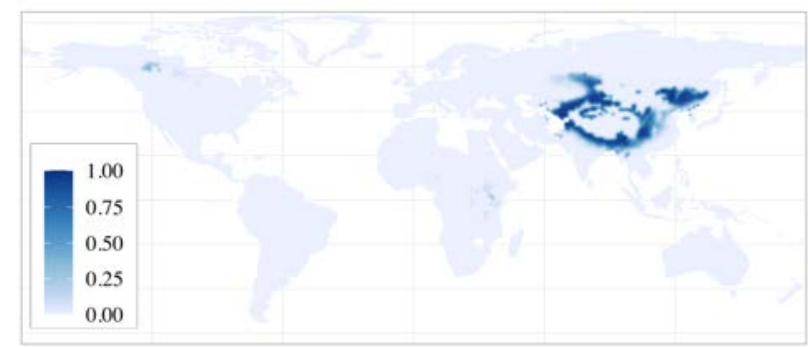

(d) Effect of climate on ag. spec.: low trade costs

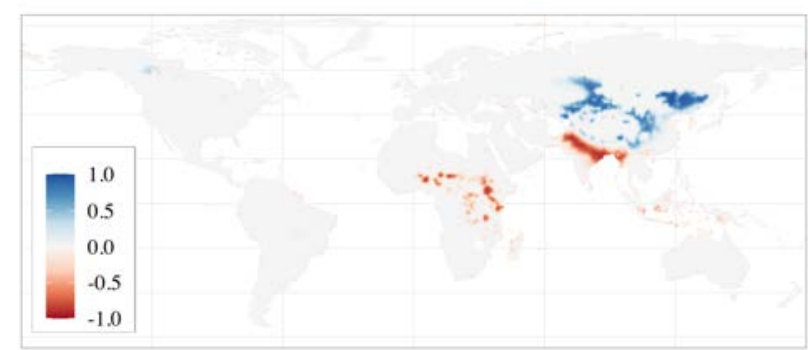

Note: Panel (a) displays share of agricultural employment under climate change with $+50 \%$ trade costs, Panel (b) displays share of agricultural employment under climate change with $-50 \%$ trade costs, Panel (c) displays difference in share of agricultural employment with and without climate change (+50\% trade costs), Panel (d) displays difference in share of agricultural employment with and without climate change (minus $\log \left(\bar{L}_{200}(r)\right)$ under no climate change with (-50\% trade costs). All maps are for period 200 .

Figure 16: Agricultural Specialization and Trade Costs

to agriculture, whereas certain places in Africa experience the opposite, switching from agriculture to nonagriculture. The Tibetan Plateau is different: starting off with much lower temperatures, the rise in temperature increases its productivity, turning it into a region that experiences an important climate-induced increase in non-agricultural output. When comparing how climate-induced changes depend on trade costs, we observe a larger drop in non-agricultural output under low trade costs in the regions of China and Central Asia where world agricultural production becomes geographically concentrated.

(a) Effect of climate on non-ag. output: high trade costs

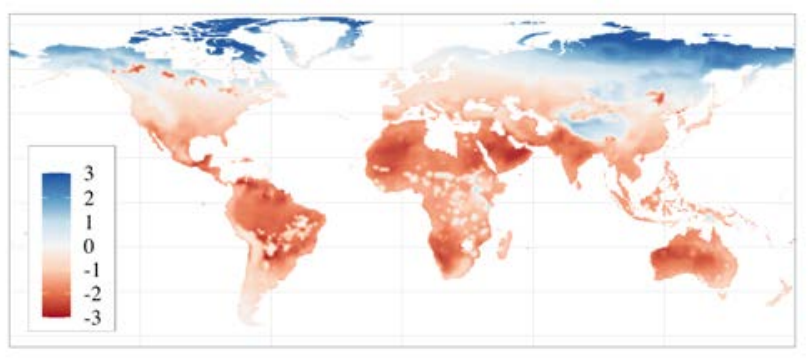

(b) Effect of climate on non-ag. output: low trade costs

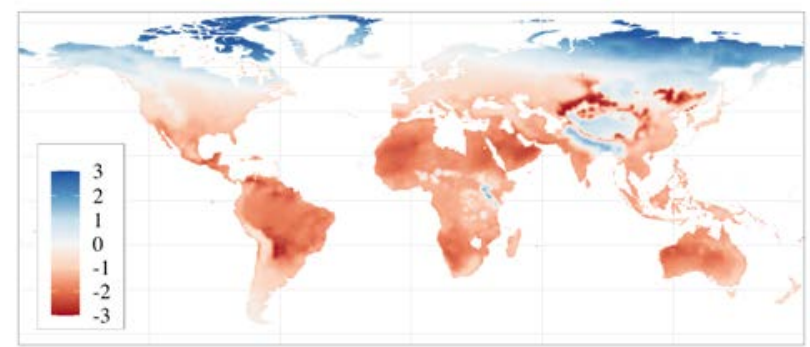

Note: Panel (a) displays log difference of non-agricultural output with and without climate change ( $+50 \%$ trade costs), Panel (b) displays log difference in non-agricultural output with and without climate change (-50\% trade costs). In both panels nonagricultural output is normalized by average nominal wages in the world. Maps are for period 200.

Figure 17: Non-Agricultural Output and Trade Costs 
Aggregate effects. By the year 2200 climate-induced losses in real GDP per capita are higher under low trade costs than under high trade costs, but this effect is reversed by the year 2400 (Figure 18 Panel (a)). On the one hand, with higher trade costs, there is less scope to respond to sector-specific climate shocks by changing specialization. This was evident from Figure 14 where we saw people adapt by migrating, rather than by shifting specialization, when trade costs are high. The lack of adaptation through trade makes a world with higher trade costs more vulnerable to climate change. On the other hand, with higher trade costs, Figure 13 showed that less people end up living in the warmest areas of the earth that suffer the most from temperature rises. This makes a world with higher trade costs less vulnerable to global warming. As Figure 18 Panel (a) shows, the second effect dominates for the first 300 years. Eventually, however, the concentration in agriculture in northern latitudes makes lower trade costs a more important advantage.

When considering utility instead of real GDP per capita, as in Figure 18 Panel (b), the losses from climate change are smaller under high trade costs than under low trade costs throughout the 400 year period under investigation (though the difference between both narrows after 200 years). This points to a greater relocation of people and economic activity toward high-amenity locations under higher trade costs.

(a) Log real GDP per capita: climate - no climate change

(b) Log utility: climate - no climate change
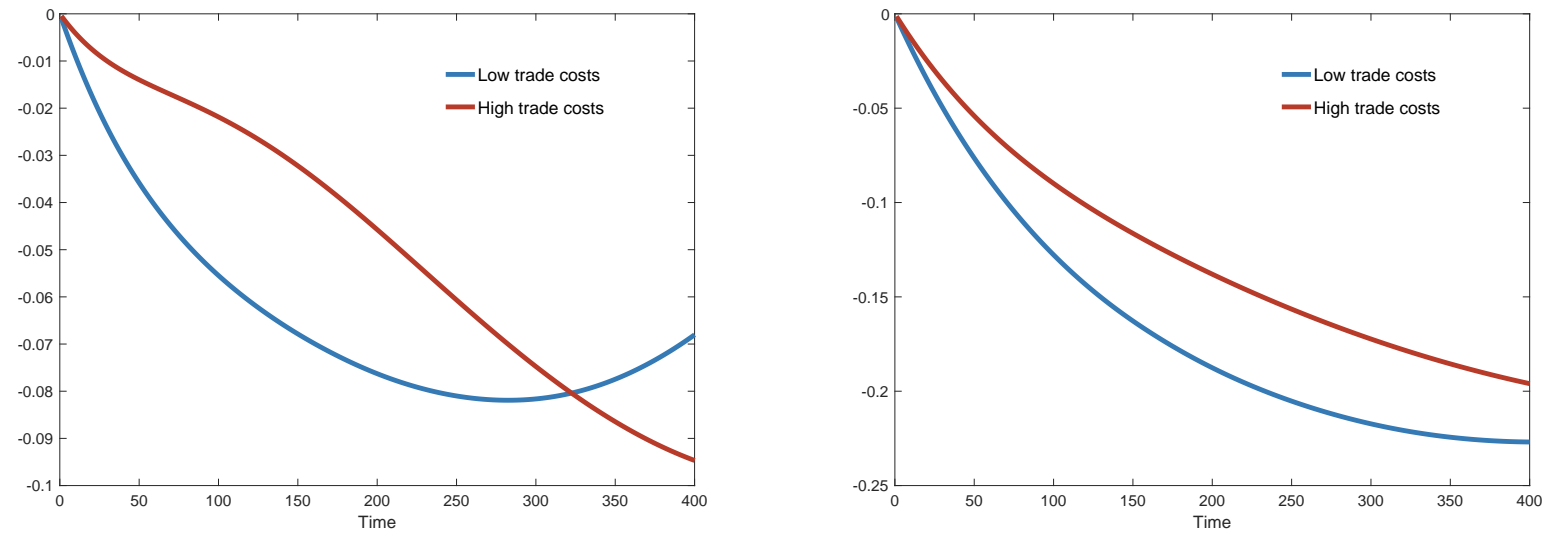

Figure 18: Aggregate Real GDP per Capita and Utility: Effect of Climate Change and Trade Costs

\section{Conclusion}

Global warming has heterogeneous effects across space, sectors, and time. Because climate shocks are location- and sector-specific, migration and trade are central to the economy's adjustment to rising temperatures. Convincingly assessing the local, sectoral, and aggregate economic effects of global warming therefore requires a multi-sector dynamic spatial model that incorporates migration and trade. This paper provides such a framework, and combines it with high-quality high-resolution data.

Under RCP 8.5, our results indicate that over a 200-year horizon rising temperatures push people and economic activity toward Siberia, Canada and Scandinavia. Because migration is costly, losses in real GDP per capita are geographically more widespread than losses in population. In a world without climate change, by the year 2200 clusters of agricultural specialization are found in Central Africa, Brazil, and India's Ganges Valley. Rising temperatures move these clusters toward Central Asia, northern Canada, and parts of China. Equatorial latitudes that suffer a relative decline in agricultural productivity fail to emerge as non- 
agricultural powerhouses. By the year 2200, predicted losses in real GDP and utility are, respectively, $6 \%$ and $15 \%$. In spite of agriculture being more sensitive to temperature than non-agriculture, global warming increases agricultural productivity growth, while it decreases non-agricultural productivity growth. This unexpected result is due to rising temperatures shifting agriculture towards regions that become highly productive once the temperature increases enough.

Higher trade costs slow down the spatial concentration of agriculture. Because goods are sourced more locally, agriculture remains closer to the world's population centers. Trade and migration are substitutes. When faced with a climate shock, the scope to adjust through changing local specialization is smaller when trade costs are high, thus increasing the incentive to move. Because migration tends to happen towards regions of relatively high productivity, higher trade costs generate smaller aggregate losses in real GDP than lower trade costs.

This paper can be extended in different directions. First, allowing for an elasticity of substitution between agriculture and non-agriculture of less than one would affect the world's vulnerability to climate change. For example, if agriculture experiences greater productivity gains than non-agriculture, it would lead to a shrinking share of employment in the sector that is most sensitive to global warming. Second, this paper has inevitably left many questions related to climate change unanswered. We have not investigated the impact of public policies, such as carbon taxes or innovation policies. Nor have we considered that climate change affects amenities. As the worlds warms, amenities in previously cold areas, such as Siberia, are bound to improve, whereas amenities in regions that already start out being very warm, will worsen. Another omission is having left out additional dimensions of climate change. Rising sea levels and more extreme weather phenomena, such as hurricanes, storm surges and droughts, are obvious examples. With adequate data, these aspects of climate change could be incorporated into the multi-sector dynamic spatial climate assessment model we have developed. 


\section{References}

Acemoglu, D., P. Aghion, L. Bursztyn, and D. Hemous (2012). The Environment and Directed Technical Change. American Economic Review 102(1), 131-166.

Acemoglu, D., U. Akcigit, D. Hanley, and W. Kerr (2016). Transition to Clean Technology. Journal of Political Economy 124(1), 52-104.

Allen, M. R., D. J. Frame, C. Huntingford, C. D. Jones, J. A. Lowe, M. Meinshausen, and N. Meinshausen (2009). Warming Caused by Cumulative Carbon Emissions towards the Trillionth Tonne. Nature 458(7242), 1163-1166.

Allen, T. and C. Arkolakis (2014). Trade and the Topography of the Spatial Economy. Quarterly Journal of Economics 129(3), 1085-1140.

Allen, T., C. Arkolakis, and X. Li (2020). On the Equilibrium Properties of Network Models with Heterogeneous Agents. Working paper, Yale University.

Balboni, C. (2019). In Harm's Way? Infrastructure Investments and the Persistence of Coastal Cities. Working Paper, MIT.

Benveniste, H., M. Oppenheimer, and M. Fleurbaey (2020). Effect of Border Policy on Exposure and Vulnerability to Climate Change. Proceedings of the National Academy of Sciences 117(43), 26692-26702.

Burzyński, M., J. de Melo, C. Deuster, and F. Docquier (2019). Climate Change, Inequality, and Human Migration. CEPR Discussion Paper 13997.

Caliendo, L., M. Dvorkin, and F. Parro (2019). Trade and Labor Market Dynamics: General Equilibrium Analysis of the China Trade Shock. Econometrica 87(3), 741-835.

Comin, D. A., M. Dmitriev, and E. Rossi-Hansberg (2012). The Spatial Diffusion of Technology. NBER Working Paper 18534.

Conte, B. (2020). Climate change and migration: the case of africa. unpublished manuscript.

Costinot, A., D. Donaldson, and C. Smith (2016). Evolving Comparative Advantage and the Impact of Climate Change in Agricultural Markets: Evidence from 1.7 Million Fields around the World. Journal of Political Economy 124(1), 205-248.

Cruz, J. L. and E. Rossi-Hansberg (2020). The Geography of Global Warming. Princeton University Working Paper.

Dell, M., B. F. Jones, and B. A. Olken (2014). What Do We Learn from the Weather? The New ClimateEconomy Literature. Journal of Economic Literature 52(3), 740-798.

Desmet, K., R. E. Kopp, S. A. Kulp, D. K. Nagy, M. Oppenheimer, E. Rossi-Hansberg, and B. H. Strauss (2020). Evaluating the Economic Cost of Coastal Flooding. American Economic Journal: Macroeconomics, forthcoming.

Desmet, K., D. K. Nagy, and E. Rossi-Hansberg (2018). The Geography of Development. Journal of Political Economy 126(3), $903-983$. 
Desmet, K. and E. Rossi-Hansberg (2014). Spatial Development. American Economic Review 104(4), 1211-1243.

Desmet, K. and E. Rossi-Hansberg (2015). On the Spatial Economic Impact of Global Warming. Journal of Urban Economics $88(\mathrm{C}), 16-37$.

Duarte, M. and D. Restuccia (2010). The Role of the Structural Transformation in Aggregate Productivity. Quarterly Journal of Economics 125(1), 129-173.

Eaton, J. and S. Kortum (2002). Technology, Geography, and Trade. Econometrica 70(5), 1741-1779.

Golosov, M., J. Hassler, P. Krusell, and A. Tsyvinski (2014). Optimal Taxes on Fossil Fuel in General Equilibrium. Econometrica 82(1), 41-88.

Hassler, J., P. Krusell, and C. Olovsson (2018). The Consequences of Uncertainty: Climate Sensitivity and Economic Sensitivity to the Climate. Annual Review of Economics 10(1), 189-205.

Hassler, J., P. Krusell, and A. Smith (2016). Environmental Macroeconomics. In J. B. Taylor and H. Uhlig (Eds.), Handbook of Macroeconomics, Volume 2B, Chapter 24, pp. 1893-2008. Elsevier.

Herrendorf, B., R. Rogerson, and Á. Valentinyi (2014). Growth and Structural Transformation. In P. Aghion and S. N. Durlauf (Eds.), Handbook of Economic Growth, Volume 2 of Handbook of Economic Growth, pp. 855 - 941. Elsevier.

IIASA and FAO (2012). Global Agro-Ecological Zones (GAEZ v3. 0).

IPCC (2020). Data Distribution Centre. Available at https://www.ipcc-data.org/.

Matthews, H. D., N. P. Gillett, P. A. Stott, and K. Zickfeld (2009). The Proportionality of Global Warming to Cumulative Carbon Emissions. Nature 459(7248), 829-832.

Nath, I. B. (2020). The Food Problem and the Aggregate Productivity Consequences of Climate Change. NBER Working Papers 27297.

Nordhaus, W. (2008). A Question of Balance: Weighing the Options on Global Warming Policies. Yale University Press.

Nordhaus, W., Q. Azam, D. Corderi Novoa, K. Hood, N. Victor, M. Mohammed, A. Miltner, and J. Weiss (2006). The G-Econ Database on Gridded Output: Methods and Data. Working Paper, Yale University.

Nordhaus, W. D. (1993). Rolling the 'DICE': An Optimal Transition Path for Controlling Greenhouse Gases. Resource and Energy Economics 15(1), 27-50.

Nordhaus, W. D. (2010). Economic Aspects of Global Warming in a Post-Copenhagen Environment. Proceedings of the National Academy of Sciences 107(26), 11721-11726.

Redding, S. J. and E. Rossi-Hansberg (2017). Quantitative spatial economics. Annual Review of Economics 9(1), 21-58.

Stocker, T. F., D. Qin, G.-K. Plattner, M. Tignor, S. K. Allen, J. Boschung, A. Nauels, Y. Xia, V. Bex, and P. M. Midgley (2013). Climate Change 2013: The Physical Science Basis. Contribution of Working Group I to the Fifth Assessment Report of the Intergovernmental Panel on Climate Change 1535. 
Świecki, T. (2017). Determinants of Structural Change. Review of Economic Dynamics 24, 95 - 131.

Uy, T., K.-M. Yi, and J. Zhang (2013). Structural Change in an Open Economy. Journal of Monetary Economics 60(6), $667-682$.

van Vuuren, D. P., J. Edmonds, M. Kainuma, K. Riahi, A. Thomson, K. Hibbard, G. C. Hurtt, T. Kram, V. Krey, J.-F. Lamarque, T. Masui, M. Meinshausen, N. Nakicenovic, S. J. Smith, and S. K. Rose (2011). The Representative Concentration Pathways: An Overview. Climatic Change 109(1), 5. 


\section{A Appendix: Solving the Model}

We start by recovering the initial distributions of fundamental agricultural productivity, $\tau_{A 0}(r)$, fundamental non-agricultural productivity, $\tau_{M 0}(r)$, and fundamental amenities relative to utility, $\bar{a}(r) / u_{0}(r)$, that rationalize the data.

First, combine equations (33) and (32) to express location $r$ 's agricultural employment as

$$
\bar{L}_{A 0}(r)=\frac{\bar{L}_{0}(r)}{1+\frac{\gamma_{M}+\mu_{M}}{\gamma_{A}+\mu_{A}}\left(\frac{Y_{0}(r)}{Y_{A 0}(r)}-1\right)}
$$

where we use the fact that $\bar{L}_{0}(r)=\bar{L}_{A 0}(r)+\bar{L}_{M 0}(r)$. Next, combining equations (33) and (36) allows us to express wages in $r$ as

$$
w_{0}(r)=\left[\gamma_{A}+\mu_{A}+\left(\gamma_{M}+\mu_{M}\right)\left(\frac{Y_{0}(r)}{Y_{A 0}(r)}-1\right)\right] \frac{Y_{A 0}(r)}{\bar{L}_{0}(r)}
$$

where we apply the normalization $\int_{S} w_{0}(r) d r=1$. Equation (17) then allows us to obtain land rents as

$$
R_{0}(r)=\frac{w_{0}(r)}{H(r)}\left(\frac{1-\gamma_{A}-\mu_{A}-\sigma_{A}}{\gamma_{A}+\mu_{A}} \bar{L}_{A 0}(r)+\frac{1-\gamma_{M}-\mu_{M}-\sigma_{M}}{\gamma_{M}+\mu_{M}} \bar{L}_{M 0}(r)\right) .
$$

Two further notes are in order. First, Cobb-Douglas sectoral shares can be identified using

$$
\frac{\chi_{A}}{\chi_{M}}=\frac{\int_{S} Y_{A 0}(r) d r}{\int_{S}\left(Y_{0}(r)-Y_{A 0}(r)\right) d r}
$$

and the fact that $\chi_{A}+\chi_{M}=1$. Second, $e_{0}$ and $\Pi_{0}$ can be obtained from equations (29) and (30), respectively.

Recover fundamental productivities and ratio of amenities to utility. With these results in hand, we are ready to recover the distribution of fundamental productivities by sector. In the case of agriculture, equation (27) implies

$$
\begin{aligned}
P_{A 0}(s)^{-\theta}= & \kappa_{A} e_{0}^{-\sigma_{A} \theta} . \\
& \int_{S} \hat{\tau}_{A 0}(r)^{\theta} w_{0}(r)^{-\left(\alpha_{A}+\gamma_{A}+\mu_{A}\right) \theta} R_{0}(r)^{\left(\alpha_{A}+\gamma_{A}+\mu_{A}+\sigma_{A}-1\right) \theta} \varsigma(s, r)^{-\theta} d r,
\end{aligned}
$$

where

$$
\hat{\tau}_{A 0}(r)=\tau_{A 0}(r) g_{A}\left(T_{0}(r)\right),
$$

while equation (28) implies

$$
\begin{aligned}
\hat{\tau}_{A 0}(r)^{-\theta}= & \chi_{A} \kappa_{A} e_{0}^{-\sigma_{A} \theta} w_{0}(r)^{-\left(\alpha_{A}+\gamma_{A}+\mu_{A}\right) \theta} R_{0}(r)^{\left(\alpha_{A}+\gamma_{A}+\mu_{A}+\sigma_{A}-1\right) \theta} Y_{A 0}(r)^{-1} . \\
& \int_{S} P_{A 0}(s)^{\theta}\left[\left(w_{0}(s)+\Pi_{0} / \bar{L}\right) \bar{L}_{0}(s)+R_{0}(s) H(s)\right] \varsigma(s, r)^{-\theta} d s
\end{aligned}
$$

In the case of non-agriculture, the same equations are

$$
\begin{aligned}
P_{M 0}(s)^{-\theta}= & \kappa_{M} e_{0}^{-\sigma_{M} \theta} . \\
& \int_{S} \hat{\tau}_{M 0}(r)^{\theta} w_{0}(r)^{-\left(\alpha_{M}+\gamma_{M}+\mu_{M}\right) \theta} R_{0}(r)^{\left(\alpha_{M}+\gamma_{M}+\mu_{M}+\sigma_{M}-1\right) \theta} \varsigma(s, r)^{-\theta} d r,
\end{aligned}
$$


where

$$
\hat{\tau}_{M 0}(r)=\tau_{M 0}(r) g_{M}\left(T_{0}(r)\right)
$$

and

$$
\begin{aligned}
\hat{\tau}_{M 0}(r)^{-\theta}= & \chi_{M} \kappa_{M} e_{0}^{-\sigma_{M} \theta} w_{0}(r)^{-\left(\alpha_{M}+\gamma_{M}+\mu_{M}\right) \theta} R_{0}(r)^{\left(\alpha_{M}+\gamma_{M}+\mu_{M}+\sigma_{M}-1\right) \theta} Y_{M 0}(r)^{-1} . \\
& \int_{S} P_{M 0}(s)^{\theta}\left[\left(w_{0}(s)+\Pi_{0} / \bar{L}\right) \bar{L}_{0}(s)+R_{0}(s) H(s)\right] \varsigma(s, r)^{-\theta} d s
\end{aligned}
$$

Our aim is to solve the system (39) and (40) for $\hat{\tau}_{A 0}(r)$ and $P_{A 0}(r)$, and solve the system (41) and (43) for $\hat{\tau}_{M 0}(r)$ and $P_{M 0}(r)$. We can show the solution to each system exists and is unique:

Lemma 1 The solution to (39) and (40) and the solution to (41) and (43) exist and both are unique to scale.

Proof. The system of (39) and (40) constitutes a special case of the systems considered in Allen, Arkolakis and $\operatorname{Li}(2020):^{14}$

$$
\prod_{h=1}^{H} x_{h}(r)^{\gamma_{k h}}=\int_{S} K_{k}(r, s) \prod_{h=1}^{H} x_{k}(r)^{\kappa_{k h}} x_{h}(s)^{\beta_{k h}} \quad k=1,2, \ldots, H
$$

such that $H=2, x_{1}(r)=P_{A 0}(r), x_{2}(r)=\hat{\tau}_{A 0}(r)$,

$$
\begin{aligned}
K_{1}(r, s)= & \kappa_{A} e_{0}^{-\sigma_{A} \theta} w_{0}(r)^{-\left(\alpha_{A}+\gamma_{A}+\mu_{A}\right) \theta} R_{0}(r)^{\left(\alpha_{A}+\gamma_{A}+\mu_{A}+\sigma_{A}-1\right) \theta} \varsigma(s, r)^{-\theta}, \\
K_{2}(r, s)= & \chi_{A} \kappa_{A} e_{0}^{-\sigma_{A} \theta} w_{0}(r)^{-\left(\alpha_{A}+\gamma_{A}+\mu_{A}\right) \theta} R_{0}(r)^{\left(\alpha_{A}+\gamma_{A}+\mu_{A}+\sigma_{A}-1\right) \theta} Y_{A 0}(r)^{-1} . \\
& {\left[\left(w_{0}(s)+\Pi_{0} / \bar{L}\right) \bar{L}_{0}(s)+R_{0}(s) H(s)\right] \varsigma(s, r)^{-\theta} }
\end{aligned}
$$

$\gamma_{11}=\gamma_{22}=-\theta, \gamma_{12}=\gamma_{21}=\kappa_{11}=\kappa_{12}=\kappa_{21}=\kappa_{22}=\beta_{11}=\beta_{22}=0$, and $\beta_{12}=\beta_{21}=\theta$. Theorem 1 in Allen, Arkolakis and Li (2020) shows that the system (44) has a unique solution (to scale) if the largest eigenvalue of the matrix $\left|\left(\mathbf{B}(\boldsymbol{\Gamma}-\mathbf{K})^{-1}\right)_{k h}\right|$ is less than or equal to one, where $\mathbf{B}$ is the matrix whose $(k, h)$ element is $\beta_{k h}, \mathbf{K}$ is the matrix whose $(k, h)$ element is $\kappa_{k h}$, and $\boldsymbol{\Gamma}$ is the matrix whose $(k, h)$ element is $\gamma_{k h}$. In our case, the largest eigenvalue of $\left|\left(\mathbf{B}(\boldsymbol{\Gamma}-\mathbf{K})^{-1}\right)_{k h}\right|$ equals one. The proof for the system of (41) and (43) is analogous.

Allen et al. (2020) also show that the solution to (44) can be found by iteration if the largest eigenvalue of $\left|\left(\mathbf{B} \boldsymbol{\Gamma}^{-1}\right)_{k h}\right|$ is strictly less than one. While this condition does not hold, we find that, in practice, iteration works on the system of (39) and (40). However, in the case of (41) and (43), iteration fails to find the solution. To address this issue, we follow the inversion procedure in Desmet et al. (2018) and approximate (41) by

$$
\begin{aligned}
P_{M 0}(s)^{-\theta}= & \kappa_{M} e_{0}^{-\sigma_{M} \theta} . \\
& \int_{S} \hat{\tau}_{M 0}(r)^{\theta-\epsilon} w_{0}(r)^{-\left(\alpha_{M}+\gamma_{M}+\mu_{M}\right) \theta} R_{0}(r)^{\left(\alpha_{M}+\gamma_{M}+\mu_{M}+\sigma_{M}-1\right) \theta} \varsigma(s, r)^{-\theta} d r
\end{aligned}
$$

where $\epsilon>0$ is a constant. For any positive $\epsilon$, the largest eigenvalue of the system of (43) and (45) is now strictly less than one, so that we can solve the system by iteration. We choose the value of $\epsilon$ sufficiently small such that the difference between equations (41) and (45) becomes negligible.

${ }^{14}$ See Remark 3 in Allen, Arkolakis and Li (2020) in particular. 
Given that agricultural and non-agricultural productivities can only be identified up to a scale, we normalize their global averages to one:

$$
\int_{S} \hat{\tau}_{A 0}(r) d r=1 \quad \text { and } \quad \int_{S} \hat{\tau}_{M 0}(r) d r=1
$$

To separate fundamental productivity $\tau_{i 0}(r)$ from $\hat{\tau}_{i 0}(r)$, we use the sector-specific temperature discount factor $g_{i}\left(T_{0}(r)\right)$. In the data section we explain how we get estimates of $g_{i}\left(T_{0}(r)\right)$.

With $w_{0}(r), R_{0}(r), P_{A 0}(r)$ and $P_{M 0}(r)$ in hand, we can express amenities relative to utility from equations (2) and (4):

$$
\frac{\bar{a}(r)}{u_{0}(r)}=\frac{P_{A 0}(r)^{\chi_{A}} P_{M 0}(r)^{\chi_{M}}}{w_{0}(r)+\Pi_{0} / \bar{L}+R_{0}(r) H(r) / \bar{L}_{0}(r)}\left(\frac{\bar{L}_{0}(r)}{H(r)}\right)^{\lambda}
$$

We have now finished solving for the initial distributions of fundamental productivities and amenities relative to utility. Since solving the system (41) and (43) required us to use an approximation, we want to ensure that this approximation is reasonable. To that end, we investigate whether the amenities and productivities backed out in the model inversion imply an equilibrium distribution of population that is sufficiently close to the one in the data. We do so by using the same algorithm as the one we use to solve the model forward.

Recovering fundamental amenities. To recover fundamental amenities $\bar{a}(r)$, we apply the same procedure as in Desmet et al. (2018). That is, we use subjective wellbeing data to measure $u_{0}(r)$ and obtain fundamental amenities as

$$
\bar{a}(r)=\frac{\bar{a}(r)}{u_{0}(r)} u_{0}(r) .
$$

We briefly discuss this in the data section.

Recovering moving costs. As in Desmet et al. (2018), we use location-level population data at time 1 to back out moving costs $m_{2}(r)$. Having total population at every location $r$ at time $1, \bar{L}_{1}(r)$, we guess a worldwide energy price $e_{1}$ as well as an agricultural employment level $\bar{L}_{A 1}(r) \in\left(0, \bar{L}_{1}(r)\right)$ and a wage $w_{1}(r)$ for every location. ${ }^{15}$ In the first step, we compute

$$
\Lambda_{1}(r)=\frac{1-\gamma_{M}-\mu_{M}-\sigma_{M}}{\gamma_{M}+\mu_{M}} \bar{L}_{1}(r)+\left(\frac{1-\gamma_{A}-\mu_{A}-\sigma_{A}}{\gamma_{A}+\mu_{A}}-\frac{1-\gamma_{M}-\mu_{M}-\sigma_{M}}{\gamma_{M}+\mu_{M}}\right) \bar{L}_{A 1}(r)
$$

and rewrite equation (17) as

$$
R_{1}(r)=w_{1}(r) \Lambda_{1}(r) H(r)^{-1} .
$$

Plugging this into equations (27) and (28), we obtain

$$
\begin{aligned}
P_{A 1}(s)^{-\theta}= & \kappa_{A} e_{1}^{-\sigma_{A} \theta} \int_{S} \tau_{A 1}(r)^{\theta} g_{A}\left(T_{1}(r)\right)^{\theta} \Lambda_{1}(r)^{\left(\alpha_{A}+\gamma_{A}+\mu_{A}+\sigma_{A}-1\right) \theta} . \\
& H(r)^{-\left(\alpha_{A}+\gamma_{A}+\mu_{A}+\sigma_{A}-1\right) \theta} w_{1}(r)^{-\left(1-\sigma_{A}\right) \theta} \varsigma(s, r)^{-\theta} d r
\end{aligned}
$$

\footnotetext{
${ }^{15}$ In practice, we start with the guesses $e_{1}=e_{0}, \bar{L}_{A 1}(r)=\bar{L}_{A 0}(r)$ and $w_{1}(r)=w_{0}(r)$.
} 


$$
\begin{gathered}
P_{M 1}(s)^{-\theta}=\kappa_{M} e_{1}^{-\sigma_{M} \theta} \int_{S} \tau_{M 1}(r)^{\theta} g_{M}\left(T_{1}(r)\right)^{\theta} \Lambda_{1}(r)^{\left(\alpha_{M}+\gamma_{M}+\mu_{M}+\sigma_{M}-1\right) \theta} . \\
H(r)^{-\left(\alpha_{M}+\gamma_{M}+\mu_{M}+\sigma_{M}-1\right) \theta} w_{1}(r)^{-\left(1-\sigma_{M}\right) \theta} \varsigma(s, r)^{-\theta} d r \\
\frac{w_{1}(r) \bar{L}_{A 1}(r)}{\gamma_{A}+\mu_{A}}=\chi_{A} \kappa_{A} e_{1}^{-\sigma_{A} \theta} \tau_{A 1}(r)^{\theta} g_{A}\left(T_{1}(r)\right)^{\theta} \Lambda_{1}(r)^{\left(\alpha_{A}+\gamma_{A}+\mu_{A}+\sigma_{A}-1\right) \theta} H(r)^{-\left(\alpha_{A}+\gamma_{A}+\mu_{A}+\sigma_{A}-1\right) \theta} . \\
w_{1}(r)^{-\left(1-\sigma_{A}\right) \theta} \int_{S} P_{A 1}(s)^{\theta}\left[\left(w_{1}(s)+\frac{\Pi_{1}}{\bar{L}}\right) \bar{L}_{1}(s)+w_{1}(s) \Lambda_{1}(s)\right] \varsigma(s, r)^{-\theta} d s
\end{gathered}
$$

and

$$
\begin{aligned}
\frac{w_{1}(r) \bar{L}_{M 1}(r)}{\gamma_{M}+\mu_{M}}= & \chi_{M} \kappa_{M} e_{1}^{-\sigma_{M} \theta} \tau_{M 1}(r)^{\theta} g_{M}\left(T_{1}(r)\right)^{\theta} \Lambda_{1}(r)^{\left(\alpha_{M}+\gamma_{M}+\mu_{M}+\sigma_{M}-1\right) \theta} H(r)^{-\left(\alpha_{M}+\gamma_{M}+\mu_{M}+\sigma_{M}-1\right) \theta} . \\
& w_{1}(r)^{-\left(1-\sigma_{M}\right) \theta} \int_{S} P_{M 1}(s)^{\theta}\left[\left(w_{1}(s)+\frac{\Pi_{1}}{\bar{L}}\right) \bar{L}_{1}(s)+w_{1}(s) \Lambda_{1}(s)\right] \varsigma(s, r)^{-\theta} d s
\end{aligned}
$$

where $\Pi_{1}$ can be obtained from equation (19). Combining (50) and (51) and rearranging yields

$$
\begin{aligned}
w_{1}(r)^{1+\theta}= & \bar{L}_{1}(r)^{-1} \int_{S}\left[\chi_{A}\left(\gamma_{A}+\mu_{A}\right) \kappa_{A} e_{1}^{-\sigma_{A} \theta} \tau_{A 1}(r)^{\theta} g_{A}\left(T_{1}(r)\right)^{\theta} \Lambda_{1}(r)^{\left(\alpha_{A}+\gamma_{A}+\mu_{A}+\sigma_{A}-1\right) \theta} .\right. \\
& H(r)^{-\left(\alpha_{A}+\gamma_{A}+\mu_{A}+\sigma_{A}-1\right) \theta} w_{1}(r)^{\sigma_{A} \theta} P_{A 1}(s)^{\theta}+\chi_{M}\left(\gamma_{M}+\mu_{M}\right) \kappa_{M} e_{1}^{-\sigma_{M} \theta} . \\
& \tau_{M 1}(r)^{\theta} g_{M}\left(T_{1}(r)\right)^{\theta} \Lambda_{1}(r)^{\left(\alpha_{M}+\gamma_{M}+\mu_{M}+\sigma_{M}-1\right) \theta} H(r)^{-\left(\alpha_{M}+\gamma_{M}+\mu_{M}+\sigma_{M}-1\right) \theta} . \\
& \left.w_{1}(r)^{\sigma_{M} \theta} P_{M 1}(s)^{\theta}\right]\left[\left(w_{1}(s)+\frac{\Pi_{1}}{\bar{L}}\right) \bar{L}_{1}(s)+w_{1}(s) \Lambda_{1}(s)\right] \varsigma(s, r)^{-\theta} d s .
\end{aligned}
$$

In the second step, we compute $P_{A 1}(s)$ and $P_{M 1}(s)$ using equations (48) and (49) and update $w_{1}(r)$ using equation (52). We proceed with this until $w_{1}(r)$ converges, where we also apply the normalization $\int_{S} w_{1}(r) d r=1$. Next, we update $\bar{L}_{A 1}(r)$ using

$$
\begin{aligned}
\bar{L}_{A 1}(r)= & \chi_{A}\left(\gamma_{A}+\mu_{A}\right) \kappa_{A} e_{1}^{-\sigma_{A} \theta} \tau_{A 1}(r)^{\theta} g_{A}\left(T_{1}(r)\right)^{\theta} \Lambda_{1}(r)^{\left(\alpha_{A}+\gamma_{A}+\mu_{A}+\sigma_{A}-1\right) \theta} H(r)^{-\left(\alpha_{A}+\gamma_{A}+\mu_{A}+\sigma_{A}-1\right) \theta} . \\
& w_{1}(r)^{-\left[1+\left(1-\sigma_{A}\right) \theta\right]} \int_{S} P_{A 1}(s)^{\theta}\left[\left(w_{1}(s)+\frac{\Pi_{1}}{\bar{L}}\right) \bar{L}_{1}(s)+w_{1}(s) \Lambda_{1}(s)\right] \varsigma(s, r)^{-\theta} d s
\end{aligned}
$$

which we obtained from rearranging equation (50), and we update $e_{1}$ using equation (29). After this, we return to the beginning of the first step. We proceed until convergence in $\bar{L}_{A 1}(r)$.

Once we know $w_{1}(r), P_{A 1}(r), P_{M 1}(r)$ and $\Lambda_{1}(r)$, we can obtain the level of utility at any location from equations (2) and (4):

$$
u_{1}(r)=\bar{a}(r)\left(\frac{\bar{L}_{1}(r)}{H(r)}\right)^{-\lambda} \frac{w_{1}(r)+\Pi_{1} / \bar{L}+w_{1}(r) \Lambda_{1}(r) / \bar{L}_{1}(r)}{P_{A 1}(r)^{\chi_{A}} P_{M 1}(r)^{\chi_{M}}}
$$

which allows us to obtain the level of moving costs (up to scale) from equation (3)

$$
m_{2}(r)=\bar{m}_{2} u_{1}(r) \bar{L}_{1}(r)^{-\Omega}
$$

where we choose the level shifter $\bar{m}_{2}$ such that $\min _{r \in S} m_{2}(r)=1$, as in Desmet et al. (2018). 
Solving for the equilibrium. We solve for the equilibrium of the model forward in time, similar to Desmet et al. (2018). As a first step, we recover the distribution of sectoral productivities in any period $t \geq 1$ by inserting the productivities and sectoral employment levels of period $t-1$ into equations (13) and (9). Next, we recover the distribution of temperature in period $t$ by substituting the carbon emissions and temperature levels of period $t-1$ into equations (20) and (22). As a final step, we solve for sectoral employment levels, wages and prices in period $t$ as a function of these productivities, so we can proceed with solving for the equilibrium of period $t+1$.

The final step of solving for the equilibrium consists of three loops embedded in each other. In the outermost loop, we guess a distribution of population $\bar{L}_{t}(r)$ and a worldwide energy price $e_{t}$, and proceed to the middle loop. ${ }^{16}$ In the middle loop, we guess a distribution of agricultural employment $\bar{L}_{A t}(r)$, compute

$$
\Lambda_{t}(r)=\frac{1-\gamma_{A}-\mu_{A}-\sigma_{A}}{\gamma_{A}+\mu_{A}} \bar{L}_{A t}(r)+\frac{1-\gamma_{M}-\mu_{M}-\sigma_{M}}{\gamma_{M}+\mu_{M}} \bar{L}_{M t}(r)
$$

and rewrite equation (17) as

$$
R_{t}(r)=w_{t}(r) \Lambda_{t}(r) H(r)^{-1}
$$

Plugging this into equations (27) and (28), we obtain

$$
\begin{gathered}
P_{A t}(s)^{-\theta}=\kappa_{A} e_{t}^{-\sigma_{A} \theta} \int_{S} \tau_{A t}(r)^{\theta} g_{A}\left(T_{t}(r)\right)^{\theta} \Lambda_{t}(r)^{\left(\alpha_{A}+\gamma_{A}+\mu_{A}+\sigma_{A}-1\right) \theta} . \\
H(r)^{-\left(\alpha_{A}+\gamma_{A}+\mu_{A}+\sigma_{A}-1\right) \theta} w_{t}(r)^{-\left(1-\sigma_{A}\right) \theta} \varsigma(s, r)^{-\theta} d r \\
P_{M t}(s)^{-\theta}=\kappa_{M} e_{t}^{-\sigma_{M} \theta} \int_{S} \tau_{M t}(r)^{\theta} g_{M}\left(T_{t}(r)\right)^{\theta} \Lambda_{t}(r)^{\left(\alpha_{M}+\gamma_{M}+\mu_{M}+\sigma_{M}-1\right) \theta} . \\
H(r)^{-\left(\alpha_{M}+\gamma_{M}+\mu_{M}+\sigma_{M}-1\right) \theta} w_{t}(r)^{-\left(1-\sigma_{M}\right) \theta} \varsigma(s, r)^{-\theta} d r \\
\frac{w_{t}(r) \bar{L}_{A t}(r)}{\gamma_{A}+\mu_{A}}=\chi_{A} \kappa_{A} e_{t}^{-\sigma_{A} \theta} \tau_{A t}(r)^{\theta} g_{A}\left(T_{t}(r)\right)^{\theta} \Lambda_{t}(r)^{\left(\alpha_{A}+\gamma_{A}+\mu_{A}+\sigma_{A}-1\right) \theta} H(r)^{-\left(\alpha_{A}+\gamma_{A}+\mu_{A}+\sigma_{A}-1\right) \theta} . \\
w_{t}(r)^{-\left(1-\sigma_{A}\right) \theta} \int_{S} P_{A t}(s)^{\theta}\left[\left(w_{t}(s)+\frac{\Pi_{t}}{\bar{L}}\right) \bar{L}_{t}(s)+w_{t}(s) \Lambda_{t}(s)\right] \varsigma(s, r)^{-\theta} d s
\end{gathered}
$$

and

$$
\begin{aligned}
\frac{w_{t}(r) \bar{L}_{M t}(r)}{\gamma_{M}+\mu_{M}}= & \chi_{M} \kappa_{M} e_{t}^{-\sigma_{M} \theta} \tau_{M t}(r)^{\theta} g_{M}\left(T_{t}(r)\right)^{\theta} \Lambda_{t}(r)^{\left(\alpha_{M}+\gamma_{M}+\mu_{M}+\sigma_{M}-1\right) \theta} H(r)^{-\left(\alpha_{M}+\gamma_{M}+\mu_{M}+\sigma_{M}-1\right) \theta} . \\
& w_{t}(r)^{-\left(1-\sigma_{M}\right) \theta} \int_{S} P_{M t}(s)^{\theta}\left[\left(w_{t}(s)+\frac{\Pi_{t}}{\bar{L}}\right) \bar{L}_{t}(s)+w_{t}(s) \Lambda_{t}(s)\right] \varsigma(s, r)^{-\theta} d s
\end{aligned}
$$

where, naturally, $\bar{L}_{M t}(r)=\bar{L}_{t}(r)-\bar{L}_{A t}(r)$, and $\Pi_{t}$ can be obtained from equation (19). Combining (58)

\footnotetext{
${ }^{16}$ In practice, we always start with guessing that the value of a given variable equals its value in period $t-1$.
} 
and (59) and rearranging yields

$$
\begin{aligned}
w_{t}(r)^{1+\theta}= & \bar{L}_{t}(r)^{-1} \int_{S}\left[\chi_{A}\left(\gamma_{A}+\mu_{A}\right) \kappa_{A} e_{t}^{-\sigma_{A} \theta} \tau_{A t}(r)^{\theta} g_{A}\left(T_{t}(r)\right)^{\theta} \Lambda_{t}(r)^{\left(\alpha_{A}+\gamma_{A}+\mu_{A}+\sigma_{A}-1\right) \theta} .\right. \\
& H(r)^{-\left(\alpha_{A}+\gamma_{A}+\mu_{A}+\sigma_{A}-1\right) \theta} w_{t}(r)^{\sigma_{A} \theta} P_{A t}(s)^{\theta}+\chi_{M}\left(\gamma_{M}+\mu_{M}\right) \kappa_{M} e_{t}^{-\sigma_{M} \theta} . \\
& \tau_{M t}(r)^{\theta} g_{M}\left(T_{t}(r)\right)^{\theta} \Lambda_{t}(r)^{\left(\alpha_{M}+\gamma_{M}+\mu_{M}+\sigma_{M}-1\right) \theta} H(r)^{-\left(\alpha_{M}+\gamma_{M}+\mu_{M}+\sigma_{M}-1\right) \theta} . \\
& \left.w_{t}(r)^{\sigma_{M} \theta} P_{M t}(s)^{\theta}\right]\left[\left(w_{t}(s)+\frac{\Pi_{t}}{\bar{L}}\right) \bar{L}_{t}(s)+w_{t}(s) \Lambda_{t}(s)\right] \varsigma(s, r)^{-\theta} d s .
\end{aligned}
$$

In the innermost loop, we guess a distribution of wages $w_{t}(r)$, and keep iterating on $w_{t}(r)$ using equation (60), also updating $P_{A t}(s)$ and $P_{M t}(s)$ using equations (56) and (57) in every iteration step. We proceed with this until convergence in $w_{t}(r)$, while we also apply the normalization $\int_{S} w_{t}(r) d r=1$. This concludes the innermost loop.

In the middle loop, we update $\bar{L}_{A t}(r)$ using

$$
\begin{aligned}
\bar{L}_{A t}(r)= & \chi_{A}\left(\gamma_{A}+\mu_{A}\right) \kappa_{A} e_{t}^{-\sigma_{A} \theta} \tau_{A t}(r)^{\theta} g_{A}\left(T_{t}(r)\right)^{\theta} \Lambda_{t}(r)^{\left(\alpha_{A}+\gamma_{A}+\mu_{A}+\sigma_{A}-1\right) \theta} H(r)^{-\left(\alpha_{A}+\gamma_{A}+\mu_{A}+\sigma_{A}-1\right) \theta} . \\
& w_{t}(r)^{-\left[1+\left(1-\sigma_{A}\right) \theta\right]} \int_{S} P_{A t}(s)^{\theta}\left[\left(w_{t}(s)+\frac{\Pi_{t}}{\bar{L}}\right) \bar{L}_{t}(s)+w_{t}(s) \Lambda_{t}(s)\right] \varsigma(s, r)^{-\theta} d s
\end{aligned}
$$

which we obtained from rearranging equation (58). We iterate on equation (61) until convergence in $\bar{L}_{A t}(r)$. This concludes the middle loop.

In the outermost loop, we update $\bar{L}_{t}(r)$ using

$$
\bar{L}_{t}(r)=\left[\digamma_{t} \frac{\left(w_{t}(r)+\Pi_{t} / \bar{L}\right) \bar{L}_{t}(r)+w_{t}(r) \Lambda_{t}(r)}{P_{A t}(r)^{\chi_{A}} P_{M t}(r)^{\chi_{M}}} \frac{\bar{a}(r)}{m_{2}(r)} H(r)^{\lambda}\right]^{\frac{1}{1+\lambda+\Omega}}
$$

which comes from combining equations (2), (3) and (4). $\digamma_{t}=\left(\frac{\bar{L}}{\int_{S} u_{t}(s)^{1 / \Omega} m_{2}(s)^{-1 / \Omega} d s}\right)^{\Omega}$ is a worldwide constant that drives the level of $\bar{L}_{t}(r)$. We do not need to explicitly solve for $\digamma_{t}$ as we can obtain the level of $\bar{L}_{t}(r)$ from the condition

$$
\int_{S} \bar{L}_{t}(r) d r=\bar{L}
$$

We iterate on equation (62) until convergence in $\bar{L}_{t}(r)$. We also update $e_{t}$ using equation (29) in every iteration step. This completes the outermost loop. 